\title{
Asymptotic behaviour of the number of Eulerian circuits
}

\author{
Mikhail Isaev * \\ Moscow Institute of Physics and Technology \\ 141700 Dolgoprudny, Russia \\ Centre de Mathématiques Appliquées, Ecole Polytechnique \\ 91128 Palaiseau, France \\ isaev.m.i@gmail.com
}

Submitted: Jun 30, 2011; Accepted: Nov 1, 2011; Published: Nov 14, 2011

Mathematics Subject Classifications: 05C30, $05 \mathrm{C} 45$

\begin{abstract}
We determine the asymptotic behaviour of the number of Eulerian circuits in undirected simple graphs with large algebraic connectivity (the second-smallest eigenvalue of the Laplacian matrix). We also prove some new properties of the Laplacian matrix.
\end{abstract}

\section{Introduction}

Let $G$ be a simple connected graph all of whose vertices have even degree. A Eulerian circuit in $G$ is a closed walk (see, for example, [2]) which uses every edge of $G$ exactly once. We let $\operatorname{Eul}(G)$ denote the number of these up to cyclic equivalence. Our purpose in this paper is to estimate $\operatorname{Eul}(G)$ for those $G$ having large algebraic connectivity.

Our method is to adopt the proof given in [6] for the case $G=K_{n}$. We refer to that paper for the interesting history of this problem, and suggest that readers who want to understand our proofs carefully may find it helpful to have a copy at hand. Since the publication of [6], the work [3] has appeared showing that counting the number of Eulerian circuits in an undirected graph is complete for the class \#P. Thus this problem is difficult in terms of complexity theory.

Here is an outline of the paper. The asymptotic formula for $\operatorname{Eul}\left(K_{n}\right)$ and our main result are presented and discussed in Section 2. In Section 3 we prove some basic properties of the Laplacian matrix, which may be of independent interest. In Section 4 we express

\footnotetext{
${ }^{*}$ This work was carried out under the supervision of S.P. Tarasov and supported in part by RFBR grant no 11-01-00398a.
} 
$\operatorname{Eul}(G)$ in terms of an $n$-dimensional integral using Cauchys formula. The value of the integral is estimated in Sections 5 and 6, using some Lemmas proved in Section 8. We prove the main result in Section 7.

\section{Asymptotic estimates of the number of Eulerian circuits}

In what follows we suppose that undirected graph $G$ has no loops and multiple edges, i.e.

$$
G \text { is a simple graph. }
$$

We also assume that

$$
\text { all vertices of } G \text { have even degrees. }
$$

Define the $n \times n$ matrix $Q$ by

$$
Q_{j k}=\left\{\begin{array}{cl}
-1, & \left(v_{j}, v_{k}\right) \in E G, \\
d_{j}, & j=k, \\
0, & \text { otherwise }
\end{array},\right.
$$

where $n=|V G|$ and $d_{j}$ is the degree of the vertex $v_{j} \in V G$. The matrix $Q=Q(G)$ is called the Laplacian matrix of the graph $G$. The eigenvalues $\lambda_{0} \leq \lambda_{1} \leq \ldots \leq \lambda_{n-1}$ of the matrix $Q$ are always non-negative real numbers and $\lambda_{0}=0$. The eigenvalue $\lambda_{1}$ is called the algebraic connectivity of the graph $G$. (For more information about the spectral properties of the Laplace matrix see, for example, [4] and [7].)

According to the Kirchhoff's Matrix-Tree-Theorem, see [5], we have that

$$
t(G)=\frac{1}{n} \lambda_{1} \lambda_{2} \cdots \lambda_{n-1},
$$

where $t(G)$ denotes the number of spanning trees of the graph $G$.

Let $p \geq 1$ be a real number and $\vec{x} \in \mathbb{R}^{n}$. We use notation

$$
\|\vec{x}\|_{p}=\left(\sum_{j=1}^{n}\left|x_{j}\right|^{p}\right)^{1 / p} .
$$

For $p=\infty$ we have the maximum norm

$$
\|\vec{x}\|_{\infty}=\max _{j}\left|x_{j}\right| .
$$

The matrix norm corresponding to the $p$-norm for vectors is

$$
\|A\|_{p}=\sup _{\vec{x} \neq 0} \frac{\|A \vec{x}\|_{p}}{\|\vec{x}\|_{p}} .
$$


We denote by $\|A\|_{H S}$ the HilbertSchmidt norm of the matrix $A$.

$$
\|A\|_{H S}=\sqrt{\sum_{j=1}^{n} \sum_{k=1}^{n}\left|A_{j k}\right|^{2}}
$$

If $f$ is bounded both above and below by $g$ asymptotically, we use the notation

$$
f(n)=\Theta_{k_{1}, k_{2}}(g(n)),
$$

which implies as $n \rightarrow \infty$, eventually

$$
k_{1}|g(n)| \leq|f(n)| \leq k_{2}|g(n)|
$$

When functions $f$ and $g$ depend not only on $n$, but also on other parameters $\vec{\xi}$, we use notation (2.9) meaning that condition (2.10) holds uniformly for all possible values of $\vec{\xi}$.

The main result of the present work is the following theorem.

Theorem 2.1. Let matrix $Q$ be the Laplacian matrix of graph $G$ with $n$ vertices. Let conditions (2.1), (2.2) hold and the algebraic connectivity $\lambda_{1} \geq \sigma n$ for some $\sigma>0$. Then as $n \rightarrow \infty$

$$
\operatorname{Eul}(G)=\Theta_{k_{1}, k_{2}}\left(2^{E-\frac{n-1}{2}} \pi^{-\frac{n-1}{2}} \sqrt{t(G)} \prod_{j=1}^{n}\left(\frac{d_{j}}{2}-1\right) !\right),
$$

where $E=|E G|, d_{j}$ is the degree of the vertex $v_{j}, t(G)$ denotes the number of spanning trees of the graph $G$ and constants $k_{1}, k_{2}>0$ depend only on $\sigma$.

Remark 2.1. We can replace condition $\lambda_{1} \geq \sigma n$ for some $\sigma>0$ in Theorem 2.1 by the condition that for some $\sigma>1 / 2$ the degree of each vertex of $G$ at least $\sigma n$.

For the complete graph $K_{n}$ one can show that $\lambda_{1}=n$ and $t\left(K_{n}\right)=n^{n-2}$.

Theorem 2.2. (variation of Theorem 4 of [6]) As $n \rightarrow \infty$ with $n$ odd

$$
\operatorname{Eul}\left(K_{n}\right)=2^{\frac{(n-1)^{2}}{2}} \pi^{-\frac{n-1}{2}} n^{\frac{n-2}{2}}\left(\left(\frac{n-1}{2}-1\right) !\right)^{n}\left(1+O\left(n^{-1 / 2+\varepsilon}\right)\right)
$$

for any $\varepsilon>0$.

In fact, Theorem 2.2 is stronger than Theorem 2.1 in the case of $G=K_{n}$. However, the asymptotic estimate of Theorem 2.1 holds for considerably broader class of graphs.

\section{Some basic properties of the Laplacian matrix}

Consider the graph $G$ such that conditions (2.1), (2.2) hold. The Laplacian matrix $Q$ has the eigenvector $[1,1, \ldots, 1]^{T}$, corresponding to the eigenvalue $\lambda_{0}=0$. We use notation $\hat{Q}=Q+J$, where $J$ denotes the matrix with every entry 1 . Note that $Q$ and $\hat{Q}$ have the 
same set of eigenvectors and eigenvalues, except for the eigenvalue corresponding to the eigenvector $[1,1, \ldots, 1]^{T}$, which equals 0 for $Q$ and $n$ for $\hat{Q}$.

Since the spectral norm is bounded above by any matrix norm we get that

$$
\lambda_{n-1}=\|Q\|_{2} \leq\|\hat{Q}\|_{2} \leq\|\hat{Q}\|_{1}=\max _{j} \sum_{k=1}^{n}\left|\hat{Q}_{j k}\right|=n .
$$

We denote by $G_{r}$ the graph which arises from $G$ by removing vertices $v_{1}, v_{2}, \ldots, v_{r}$ and all adjacent edges.

Lemma 3.1. Let condition (2.1) holds for graph $G$ with $n$ vertices. Then

$$
\begin{gathered}
\lambda_{1}(G) \leq \frac{n}{n-1} \min _{j} d_{j}, \\
\lambda_{1}(G) \geq 2 \min _{j} d_{j}-n+2, \\
\lambda_{1}\left(G_{r}\right) \geq \lambda_{1}(G)-r,
\end{gathered}
$$

where $\lambda_{1}(G)$ is the algebraic connectivity of $G$ and $d_{j}$ is the degree of the vertex $v_{j} \in V G$.

The proof of Lemma 3.1 can be found in [4].

Lemma 3.2. Let condition (2.1) hold and the algebraic connectivity $\lambda_{1} \geq \sigma n$ for some $\sigma>0$. Then there is a constant $c_{\infty}>0$ depending only on $\sigma$ such that

$$
\left\|\hat{Q}^{-1}\right\|_{1}=\left\|\hat{Q}^{-1}\right\|_{\infty} \leq \frac{c_{\infty}}{n}
$$

Proof of Lemma 3.2. We consider $\vec{x} \in \mathbb{R}^{n}$ such that $\|\vec{x}\|_{\infty}=1$. For simplicity, we assume that $\left|x_{1}\right|=1$. We denote by $J_{\sigma}$ the set of the indices $j$ such that $\left|x_{j}\right| \geq \sigma / 8$.

In the case of $\left|J_{\sigma}\right| \geq \sigma n / 4$ we have that

$$
\|\vec{x}\|_{2} \geq \sqrt{\frac{\sigma^{2}}{64} \sigma n / 4}
$$

Since the algebraic connectivity $\lambda_{1} \geq \sigma n$, we get that

$$
\sqrt{n\|\hat{Q} \vec{x}\|_{\infty}^{2}} \geq\|\hat{Q} \vec{x}\|_{2} \geq \lambda_{1}\|\vec{x}\|_{2} \geq \sigma n\|\vec{x}\|_{2} \geq \sigma n \sqrt{\frac{\sigma^{3} n}{256}}
$$

In the case of $\left|J_{\sigma}\right| \leq \sigma n / 4$ we have that

$$
\begin{aligned}
\|\hat{Q} \vec{x}\|_{\infty} & \geq\left(d_{1}+1\right)\left|x_{1}\right|-\sum_{j=2}^{n}\left|x_{j}\right| \\
& \geq d_{1}+1-\sum_{j \in J_{\sigma}}\left|x_{j}\right|-\sum_{j \notin J_{\sigma}}\left|x_{j}\right| \\
& \geq d_{1}+1-\sigma n / 4-n \sigma / 8 .
\end{aligned}
$$


Using again $\lambda_{1} \geq \sigma n$ and (3.2) we get that

$$
d_{1} \geq \min _{j} d_{j} \geq \frac{n-1}{n} \sigma n \geq \sigma n / 2 .
$$

Combining (3.7), (3.8) and (3.9) we obtain that

$$
\|\hat{Q} \vec{x}\|_{\infty} \geq c_{\infty}^{-1} n\|\vec{x}\|_{\infty}
$$

for some constant $c_{\infty}>0$ depending only on $\sigma$.

The following lemmas will be applied to estimate the determinant of a matrix close to the identity matrix $I$.

Lemma 3.3. Let $X$ be an $n \times n$ matrix such that $\|X\|_{2}<1$. Then for fixed $m \geq 2$

$$
\operatorname{det}(I+X)=\exp \left(\sum_{r=1}^{m-1} \frac{(-1)^{r+1}}{r} \operatorname{tr}\left(X^{r}\right)+E_{m}(X)\right)
$$

where $\operatorname{tr}$ is the trace function and

$$
\left|E_{m}(X)\right| \leq \frac{n}{m} \frac{\|X\|_{2}^{m}}{1-\|X\|_{2}}
$$

Lemma 3.3 was also formulated and proved in [6].

Lemma 3.4. Let the assumptions of Lemma 3.3 hold and all eigenvalues of $X$ are nonnegative real numbers. Then

$$
\operatorname{det}(I-X) \geq \exp \left(-\frac{\operatorname{tr}(X)}{1-\|X\|_{2}}\right) .
$$

Proof of Lemma 3.4. Using Lemma 3.3 we get that

$$
\operatorname{det}(I-X)=\exp \left(\sum_{r=1}^{\infty} \frac{(-1)^{r+1}}{r}(-1)^{r} \operatorname{tr}\left(X^{r}\right)\right)
$$

Since all eigenvalues of $X$ are non-negative real numbers

$$
0 \leq \operatorname{tr}\left(X^{r}\right) \leq \operatorname{tr}(X)\|X\|_{2}^{r-1}
$$

Hence

$$
\operatorname{det}(I-X) \geq \exp \left(-\sum_{r=1}^{\infty} \frac{\operatorname{tr}(X)}{r}\|X\|_{2}^{r-1}\right) \geq \exp \left(-\frac{\operatorname{tr}(X)}{1-\|X\|_{2}}\right)
$$


Lemma 3.5. Let the assumptions of Lemma 3.2 hold. Then there is a constant $c_{1}>0$ depending only on $\sigma$ such that

$$
\left|\operatorname{det} M_{11}\right| \leq c_{1} \frac{\operatorname{det} \hat{Q}}{n}
$$

where $M_{11}$ denotes the $(n-1) \times(n-1)$ matrix that results from deleting the first row and the first column of $\hat{Q}=Q+J$.

Proof of Lemma 3.5. Since the algebraic connectivity $\lambda_{1} \geq \sigma n$, using (3.2), we get the following estimate for the degree $d_{k}$ of the vertex $v_{k} \in V G$.

$$
d_{k} \geq \min _{j} d_{j} \geq \frac{n-1}{n} \sigma n \geq \sigma n / 2 .
$$

Consider the $n \times n$ matrix $X$ such that

$$
X_{j k}=\left\{\begin{array}{cl}
\frac{1}{d_{1}+1}, & \text { if }\left(v_{1}, v_{j}\right) \notin E G,\left(v_{1}, v_{k}\right) \notin E G \text { and } j, k \neq 1, \\
0, & \text { otherwise. }
\end{array}\right.
$$

After performing one step of the Gaussian elimination for $\hat{Q}+X$, we obtain that

$$
\operatorname{det}(\hat{Q}+X)=\left(d_{1}+1\right) \operatorname{det} M_{11}
$$

Since the spectral norm is bounded above by any matrix norm, we get that

$$
\|X\|_{2} \leq\|X\|_{1} \leq \frac{n}{d_{1}+1} \leq \frac{2}{\sigma}
$$

Since $\lambda_{1} \geq \sigma n$, taking into account (3.1), we obtain that

$$
\left\|X \hat{Q}^{-1}\right\|_{2} \leq\|X\|_{2}\left\|\hat{Q}^{-1}\right\|_{2} \leq \frac{2}{\sigma \lambda_{1}} \leq \frac{2}{\sigma^{2} n}
$$

Combining Lemma 3.3 with (3.22), we get that as $n \rightarrow \infty$

$$
\operatorname{det}\left(I+X \hat{Q}^{-1}\right)=\exp \left(\operatorname{tr}\left(X \hat{Q}^{-1}\right)+E_{2}\left(X \hat{Q}^{-1}\right)\right) \leq \exp \left(n \frac{2}{\sigma^{2} n}+O\left(n^{-1}\right)\right)
$$

From (3.20) and (3.23) we have that as $n \rightarrow \infty$

$$
\left(d_{1}+1\right) \operatorname{det} M_{11}=\operatorname{det}\left(I+X \hat{Q}^{-1}\right) \operatorname{det} \hat{Q} \leq \operatorname{det} \hat{Q} \exp \left(2 / \sigma^{2}+O\left(n^{-1}\right)\right) .
$$

Since $\hat{Q}$ is positive definite, using (3.18) in (3.24), we obtain (3.17). 
Lemma 3.6. Let the assumptions of Lemma 3.2 hold. Let $G_{r}$ be the graph which arises from $G$ by removing vertices $v_{1}, v_{2}, \ldots, v_{r}$ and all adjacent edges. Then there is a constant $c_{2}>0$ depending only on $\sigma$ such that for any $\varepsilon \in(0,1)$ and $r \leq n^{\varepsilon}$

$$
\operatorname{det} \hat{Q}\left(G_{r}\right) \geq \frac{\operatorname{det} \hat{Q}(G)}{\left(c_{2} n\right)^{r}} .
$$

Proof of Lemma 3.6. We give first a proof for the case of $r=1$. For our purpose it is convenient to use notations $\hat{Q}=\hat{Q}(G)$ and $\hat{Q}_{1}=\hat{Q}\left(G_{1}\right)$. Note that the matrix $M_{11}$ that results from deleting the first row and the first column of $\hat{Q}$ coincides with the matrix $\hat{Q}_{1}$ with the exception of the diagonal elements. In a similar way as (3.20) we get that

$$
\operatorname{det}(\hat{Q}+\Omega+X)=\left(d_{1}+1\right) \operatorname{det} \hat{Q}_{1},
$$

where $X$ is such that

$$
X_{j k}=\left\{\begin{array}{cl}
\frac{1}{d_{1}+1}, & \text { if }\left(v_{1}, v_{j}\right) \notin E G,\left(v_{1}, v_{k}\right) \notin E G \text { and } j, k \neq 1, \\
0, & \text { otherwise. }
\end{array}\right.
$$

and $\Omega$ is such diagonal matrix that

$$
\Omega_{j j}= \begin{cases}1, & \text { if }\left(v_{1}, v_{j}\right) \in E G \\ 0, & \text { otherwise }\end{cases}
$$

Taking into account (3.21), we have that

$$
\|\Omega+X\|_{2} \leq\|\Omega\|_{2}+\|X\|_{2} \leq 1+\frac{2}{\sigma} \leq \frac{3}{\sigma} .
$$

In a similar way as (3.22) we get that

$$
\left\|(\Omega+X) \hat{Q}^{-1}\right\|_{2} \leq\|\Omega+X\|_{2}\left\|\hat{Q}^{-1}\right\|_{2} \leq \frac{3}{\sigma \lambda_{1}} \leq \frac{3}{\sigma^{2} n}
$$

Combining Lemma 3.3 with (3.30), we get that as $n \rightarrow \infty$

$$
\begin{aligned}
\operatorname{det}\left(I+(\Omega+X) \hat{Q}^{-1}\right) & =\exp \left(\operatorname{tr}\left((\Omega+X) \hat{Q}^{-1}\right)+E_{2}\left((\Omega+X) \hat{Q}^{-1}\right)\right) \\
\geq & \exp \left(-n \frac{3}{\sigma^{2} n}+O\left(n^{-1}\right)\right) .
\end{aligned}
$$

From (3.26) and (3.31) we have that as $n \rightarrow \infty$

$$
\left(d_{1}+1\right) \operatorname{det} \hat{Q}_{1}=\operatorname{det}\left(I+(\Omega+X) \hat{Q}^{-1}\right) \operatorname{det} \hat{Q} \geq \operatorname{det} \hat{Q} \exp \left(-3 / \sigma^{2}+O\left(n^{-1}\right)\right) .
$$

Since $d_{1}+1 \leq n$ we get (3.25) for the case of $r=1$.

Taking into account (3.4) and using $r$ times (3.32) we get (3.25) for the general case. 
According to (2.4), we have that

$$
t(G)=\frac{1}{n} \lambda_{1} \lambda_{2} \cdots \lambda_{n-1}=\frac{\operatorname{det} \hat{Q}}{n^{2}},
$$

where $t(G)$ denotes the number of spanning trees of the graph $G$.

Lemma 3.7. Let the assumptions of Lemma 3.2 hold. Then for some $c_{3}>0$ depending only on $\sigma$ the number of spanning trees of the graph $G$ with maximum degree greater than $d$ is less then $c_{3}^{n} \operatorname{det} \hat{Q} / d$ ! for all $d \geq 0$.

Proof of Lemma 3.7. According to Lemma 5 of [6] the number of labelled trees on $n$ vertices with first vertex having degree greater than $d$ is less than $2 n^{n-2} / d$ ! for all $d \geq 0$. We have that

$$
\operatorname{det} \hat{Q} \geq \lambda_{1}^{n} \geq(\sigma n)^{n} .
$$

To complete proof it remains to note that the number of spanning trees with maximum degree greater than $d$ in $G$ does not exceed the number of such spanning trees in the complete graph with $n$ vertices.

Consider a spanning tree $T$ of the graph $G$. We denote by $G_{T}$ the graph which arises from $G$ by removing all edges of the tree $T$.

Lemma 3.8. Let the assumptions of Lemma 3.2 hold. Let $T$ be the spanning tree of $G$ with the maximum degree at most $\sigma n / 4$. Then the algebraic connectivity $\lambda_{1}\left(G_{T}\right) \geq \sigma n / 2$ and

$$
\operatorname{det} \hat{Q}\left(G_{T}\right) \geq c_{4} \operatorname{det} \hat{Q}(G)
$$

for some $c_{4}>0$ depending only on $\sigma$.

Proof of Lemma 3.8. Note that

$$
\hat{Q}\left(G_{T}\right)=\hat{Q}(G)-Q(T) .
$$

Since the spectral norm is bounded above by any matrix norm and the maximum degree of vertex of $T$ at most $\sigma n / 4$ we get that

$$
\|Q(T)\|_{2} \leq\|Q(T)\|_{1} \leq \sigma n / 2 .
$$

Therefore, since the algebraic connectivity $\lambda_{1}(G) \geq \sigma n$, we have that

$$
\lambda_{1}\left(G_{T}\right) \geq \lambda_{1}(G)-\|Q(T)\|_{2} \geq \sigma n / 2
$$

and

$$
\operatorname{det} \hat{Q}\left(G_{T}\right)=\operatorname{det} \hat{Q}(G) \operatorname{det}(I-X),
$$

where $X=Q(T) \hat{Q}(G)^{-1}$. Note that $\hat{Q}(G)$ is the matrix of positive definite quadratic form and $Q(T)$ is the matrix of quadratic form with non-negative eigenvalues. Considering the basis in which both matrices are diagonal, we have that

$$
\operatorname{tr}\left(Q(T) \hat{Q}(G)^{-1}\right) \leq \operatorname{tr}(Q(T))\left\|\hat{Q}(G)^{-1}\right\|_{2}
$$


and

$$
\text { all eigenvalues of } Q(T) \hat{Q}(G)^{-1} \text { are non-negative. }
$$

Using again the fact that the algebraic connectivity $\lambda_{1}(G) \geq \sigma n$ and (3.37) we get that

$$
\|X\|_{2} \leq\|Q(T)\|_{2}\left\|\hat{Q}(G)^{-1}\right\|_{2} \leq \frac{\sigma n}{2} \frac{1}{\sigma n}=\frac{1}{2} .
$$

Since $T$ is the spanning tree $\operatorname{tr}(Q(T))=2(n-1)$. Using $(3.40)$, we get that

$$
\operatorname{tr}(X) \leq \operatorname{tr}(Q(T))\left\|\hat{Q}(G)^{-1}\right\|_{2} \leq 2 n \frac{1}{\sigma n}=\frac{2}{\sigma} .
$$

To complete the proof it remains to combine Lemma 3.4 with (3.39), (3.42) and (3.43).

Lemma 3.9. Let $a>0$ and the assumptions of Lemma 3.2 hold. Then for any set $A \subset V G$ such, that $|A| \geq$ an, there is a function $h: V G \rightarrow \mathbb{N}_{0}$, having following properties:

$$
\begin{gathered}
h(v)=0, \text { if } v \in A, \quad h(v) \leq H, \text { for any } v \in V G, \\
\mid\{w \in V G \mid(w, v) \in E G \text { and } h(w)<h(v)\} \mid \geq \alpha n \text {, if } v \notin A,
\end{gathered}
$$

where constants $H, \alpha>0$ depend only on a and $\sigma$.

Proof of Lemma 3.9. At first, we construct the set $A_{1}=\{v \in V G \mid h(v)=1\}$, having property (3.45).

If $|A|>n-\sigma n / 4$, then let $A_{1}=\{v \in V G \mid v \notin A\}$. Taking into account (3.18), we get that property (3.45) hold for $\alpha=\sigma / 4$. In this case $H=1$.

For $|A| \leq n-\sigma n / 4$ define $\vec{x} \in \mathbb{R}^{n}$ such that

$$
x_{j}=\left\{\begin{array}{cc}
1-|A| / n, & v_{j} \in A, \\
-|A| / n, & v_{j} \notin A .
\end{array}\right.
$$

Since $x_{1}+x_{2}+\ldots+x_{n}=0$

$$
\vec{x}^{T} Q \vec{x}=\vec{x}^{T} \hat{Q} \vec{x} \geq \lambda_{1}\|\vec{x}\|_{2}^{2} \geq \lambda_{1}|A|\left(\frac{n-|A|}{n}\right)^{2} \geq \sigma n \text { an }(\sigma / 4)^{2}=\frac{a \sigma^{3} n^{2}}{16} .
$$

On the other hand,

$$
\vec{x}^{T} Q \vec{x}=\sum_{\left(v_{j}, v_{k}\right) \in E G}\left(x_{j}-x_{k}\right)^{2},
$$

which is equal to the number of edges $(v, w) \in E G$, where $v \in A, w \notin A$. We denote $A_{1}$ the set of vertices $w \notin A$, having at least $\alpha n$ adjacent vertices in $A$, where $\alpha=\frac{1}{32} a \sigma^{3}$.

$$
\vec{x}^{T} Q \vec{x} \leq n\left|A_{1}\right|+\alpha n|V G| .
$$

Combining (3.47) and (3.49), we get that $\left|A_{1}\right| \geq \alpha n$.

We make further construction of the function $h$ inductively, using for the $k$-th step the set $A^{(k)}=A \cup A_{1} \cup \ldots \cup A_{k}$. The number of steps does not exceed $1 / \alpha$ as $\left|A_{k}\right| \geq \alpha n$ for each step, perhaps with the exception of the last one. 


\section{The result expressed as an integral}

The reasoning below is similar to the arguments of Section 2 of [6]

An Eulerian orientation of $G$ is an orientation of its edges with the property that for every vertex both the in-degree and the out-degree are equal. Any Eulerian circuit induces an Eulerian orientation by orienting each edge in accordance with its direction of traversal.

A directed tree with root $v$ is a connected directed graph $T$ such that $v \in V T$ has outdegree zero, and each other vertex has out-degree one. Thus, $T$ is a tree which has each edge oriented towards $v$.

Let $D$ be a directed graph with $n$ vertices, and let $v \in V D$. A directed spanning tree of $D$ with root $v$ is a spanning subgraph of $D$ which is a directed tree with root $v$.

The following famous theorem, sometimes called the BEST Theorem, is due to de Bruijn, van Aardenne-Ehrenfest, Smith, and Tutte [1,8].

Theorem 4.1. Let $D$ be a directed graph with vertices $v_{1}, v_{2}, \ldots, v_{n}$. Suppose that there are numbers $d_{1}, d_{2}, \ldots, d_{n}$ such that, for every vertex $v_{r}$, both the in-degree and the outdegree of $v_{r}$ are equal to $d_{r}$. Let $t_{r}=t_{r}(D)$ be the number of directed spanning trees of $D$ rooted at $v_{r}$. Then $t_{r}$ is independent of $r$, and

$$
\operatorname{Eul}(D)=t_{r} \prod_{j=1}^{n}\left(d_{j}-1\right) !
$$

Consider the undirected graph $G$ with $n$ vertices such that conditions (2.1), (2.2) hold. Note that for every spanning tree $T$ of the graph $G$ and any vertex $v_{r} \in V G$ there is only one orientation of the edges of $T$ such that we obtain a directed tree with root $v_{r}$. We

denote by $\mathcal{T}_{r}$ the set of directed trees with root $v_{r}$ obtained in such a way. For $T \in \bigcup_{r=1}^{n} \mathcal{T}_{r}$ denote by $E O(T)$ the number of Eulerian orientations of $G$ that the corresponding graphs contain $T$.

From Theorem 4.1 in the case of a graph $D$ corresponding to Eulerian orientation of the graph $G$ we find that

$$
\operatorname{Eul}(D)=t_{r}(D) \prod_{j=1}^{n}\left(\frac{d_{j}}{2}-1\right) !
$$

where $d_{j}$ is the degree of the vertex $v_{j} \in V G$. Let denote by $\mathcal{E} \mathcal{O}$ the set of all graphs corresponding to Eulerian orientations of the graph $G$. Grouping Eulerian circuits according to the induced orientations, we obtain that

$$
\operatorname{Eul}(G)=\sum_{D \in \mathcal{E} \mathcal{O}} \operatorname{Eul}(D)=\prod_{j=1}^{n}\left(\frac{d_{j}}{2}-1\right) ! \sum_{D \in \mathcal{E} \mathcal{O}} t_{r}(D)
$$

for any fixed natural number $r \leq n$. 
Regrouping the terms of the final summation according to the directed subtrees rooted at $v_{r}$, we find that

$$
\operatorname{Eul}(G)=\prod_{j=1}^{n}\left(\frac{d_{j}}{2}-1\right) ! \sum_{T \in \mathcal{T}_{r}} E O(T) .
$$

For $n \geq 1$ and $R \geq 0$ we use notation $U_{n}(R)=\left\{\left(x_{1}, x_{2}, \ldots, x_{n}\right)|| x_{i} \mid<R\right.$ for all $\left.i\right\}$. The value of $E O(T)$ is the constant term in

$$
\prod_{\left(v_{j}, v_{k}\right) \in E G}\left(x_{j}^{-1} x_{k}+x_{k}^{-1} x_{j}\right) \prod_{\left(v_{j}, v_{k}\right) \in E T} \frac{x_{k}^{-1} x_{j}}{\left(x_{j}^{-1} x_{k}+x_{k}^{-1} x_{j}\right)},
$$

which we can extract via Cauchys Theorem using the unit circle as a contour for each variable. Making the substitution $x_{j}=e^{i \theta_{j}}$ for each $j$, we find that

$$
\operatorname{Eul}(G)=\prod_{j=1}^{n}\left(\frac{d_{j}}{2}-1\right) ! 2^{|E G|-n+1} \pi^{-n} S
$$

where

$$
S=\int_{U_{n}(\pi / 2)} \prod_{\left(v_{j}, v_{k}\right) \in E G} \cos \Delta_{j k} \sum_{T \in \mathcal{T}_{r}} \prod_{\left(v_{j}, v_{k}\right) \in E T}\left(1+i \tan \Delta_{j k}\right) d \vec{\theta},
$$

having put $\Delta_{j k}=\theta_{j}-\theta_{k}$ and using the fact that the integrand is unchanged by the substitutions $\theta_{j} \rightarrow \theta_{j}+\pi$ if condition (2.2) holds.

We approach the integral by first estimating it in the region that would turn out to be the asymptotically significant one. Then we bound the integral over the remaining regions and show that it is vanishingly small in comparison with the significant part.

\section{The dominant part of the integral}

In what follows, we fix some small constant $\varepsilon>0$. Define

$$
V_{0}=\left\{\vec{\theta} \in U_{n}(\pi / 2):\left|\theta_{j}-\bar{\theta}\right|(\bmod \pi) \leq n^{-1 / 2+\varepsilon}, \text { where } \bar{\theta}=\frac{\theta_{1}+\ldots+\theta_{n}}{n}\right\}
$$

and let $S_{0}$ denote the contribution to $S$ of $\vec{\theta} \in V_{0}$. Since the integrand is invariant under uniform translation of all the $\theta_{j}$ 's $\bmod \pi$, we can fix $\bar{\theta}=0$ and multiply it by the ratio of its range $\pi$ to the length $n^{-1 / 2}$ of the vector $\frac{1}{n}[1,1, \ldots, 1]^{T}$. Thus we have that

$$
S_{0}=\pi n^{1 / 2} \int_{L \cap V_{0}} \prod_{\left(v_{j}, v_{k}\right) \in E G} \cos \Delta_{j k} \sum_{T \in \mathcal{T}_{r}} \prod_{\left(v_{j}, v_{k}\right) \in E T}\left(1+i \tan \Delta_{j k}\right) d L
$$

where $L$ denotes the orthogonal complement to the vector $[1,1, \ldots, 1]^{T}$.

The sum over $\mathcal{T}_{r}$ in the integrand of (5.2) can be expressed as a determinant, according to the following theorem of [9]. 
Theorem 5.1. Let $w_{j k}(1 \leq j, k \leq n, j \neq k)$ be arbitrary. Define the $n \times n$ matrix $A$ by

$$
A_{j k}=\left\{\begin{array}{cc}
-w_{j k}, & \text { if } j \neq k, \\
\sum_{r \neq j} w_{j r}, & \text { if } k=j
\end{array}\right.
$$

the sum being over $1 \leq r \leq n$ with $r \neq j$. For any $r$ with $1 \leq r \leq n$, let $M_{r}$ denote the principal minor of $A$ formed by removing row $r$ and column $r$. Then

$$
\operatorname{det} M_{r}=\sum_{T} \prod_{\left(v_{j}, v_{k}\right) \in E T} w_{j k}
$$

where the sum is over all directed trees $T$ with $V T=\left\{v_{1}, v_{2}, \ldots, v_{n}\right\}$ and root $v_{r}$.

Lemma 5.1. Let the assumptions of Theorem 2.1 hold. Let $\hat{Q}=Q+J$, where J denotes the matrix with every entry 1 . Then for $\vec{\theta} \in V_{0}$ as $n \rightarrow \infty$

$$
\sum_{r=1}^{n} \sum_{T \in \mathcal{T}_{r}} \prod_{\left(v_{j}, v_{k}\right) \in E T}\left(1+i \tan \Delta_{j k}\right)=\frac{e^{i \vec{\theta}^{T} Q \vec{\alpha}+\operatorname{tr}\left(\Lambda \hat{Q}^{-1}\right)^{2}}}{n} \operatorname{det} \hat{Q}\left(1+O\left(n^{-1 / 2+3 \varepsilon}\right)\right),
$$

where $\vec{\alpha}$ denotes the vector composed of the diagonal elements of the matrix $\hat{Q}^{-1}, \Lambda$ denotes the diagonal matrix whose diagonal elements are equal to the components of the vector $Q \vec{\theta}$.

Proof of Lemma 5.1. Define the $n \times n$ matrix $B$ by

$$
B_{j k}= \begin{cases}-\tan \Delta_{j k}, & \text { for }\left(v_{j}, v_{k}\right) \in E G, \\ \sum_{l:\left(v_{j}, v_{l}\right) \in E G} \tan \Delta_{j l}, & \text { for } k=j, \\ 0 & \text { otherwise }\end{cases}
$$

Using Theorem 5.1 with the matrix $A=Q+i B$, we get that

$$
\sum_{r=1}^{n} \sum_{T \in \mathcal{T}_{r}} \prod_{\left(v_{j}, v_{k}\right) \in E T}\left(1+i \tan \Delta_{j k}\right)=\sum_{r=1}^{n} M_{r}
$$

where $M_{r}$ denotes the principal minor of $A$ formed by removing row $r$ and column $r$. Since the vector $[1,1, \ldots, 1]^{T}$ is the common eigenvector of the matrices $Q$ and $B$, corresponding to the eigenvalue 0 , we find that

$$
\sum_{r=1}^{n} M_{r}=\frac{\operatorname{det}(\hat{Q}+i B)}{n} .
$$

Note that for $\vec{\theta} \in V_{0}$

$$
\Delta_{j k}=\left(\theta_{j}-\bar{\theta}\right)-\left(\theta_{k}-\bar{\theta}\right) \leq 2 n^{-1 / 2+\varepsilon} .
$$

Since the spectral norm is bounded above by any matrix norm we get that

$$
\|B\|_{2} \leq\|B\|_{1}=\max _{j} \sum_{k=1}^{n}\left|B_{j k}\right|=O\left(n^{1 / 2+\varepsilon}\right) .
$$


Let $\Phi=B \hat{Q}^{-1}$. Since the algebraic connectivity $\lambda_{1} \geq \sigma n$, we get that

$$
\|\Phi\|_{2} \leq\|B\|_{2}\left\|\hat{Q}^{-1}\right\|_{2} \leq \frac{1}{\lambda_{1}}\|B\|_{2}=O\left(n^{-1 / 2+\varepsilon}\right) .
$$

Using Lemma (3.3) with the matrix $i \Phi$, we find that as $n \rightarrow \infty$

$$
\operatorname{det}(I+i \Phi)=\exp \left(\operatorname{tr}(i \Phi)+\frac{\operatorname{tr}\left(\Phi^{2}\right)}{2}+O\left(n^{-1 / 2+3 \varepsilon}\right)\right)
$$

Let

$$
B=B_{\text {skew }}+B_{\text {diag }}
$$

where $B_{\text {skew }}$ is the skew-symmetric matrix and $B_{\text {diag }}$ is the diagonal matrix. Since $\hat{Q}$ is the symmetric matrix

$$
\operatorname{tr}\left(B_{\text {skew }} \hat{Q}^{-1}\right)=0 .
$$

Using (5.9), note that as $n \rightarrow \infty$

$$
\left\|B_{\text {diag }}-\Lambda\right\|_{2}=O\left(n^{-1 / 2+3 \varepsilon}\right)
$$

where $\Lambda$ denotes the diagonal matrix whose diagonal elements are equal to the components of the vector $Q \vec{\theta}$. Since the algebraic connectivity $\lambda_{1} \geq \sigma n$, we get that as $n \rightarrow \infty$

$$
\left|\operatorname{tr}\left(\left(B_{\text {diag }}-\Lambda\right) \hat{Q}^{-1}\right)\right| \leq n\left\|B_{\text {diag }}-\Lambda\right\|_{2}\left\|\hat{Q}^{-1}\right\|_{2}=O\left(n^{-1 / 2+3 \varepsilon}\right) .
$$

Using (5.14) and (5.16), we obtain that as $n \rightarrow \infty$

$$
\operatorname{tr}(\Phi)=\operatorname{tr}\left(B_{\text {diag }} \hat{Q}^{-1}\right)=\operatorname{tr}\left(\Lambda \hat{Q}^{-1}\right)+O\left(n^{-1 / 2+3 \varepsilon}\right)=\vec{\theta}^{T} Q \vec{\alpha}+O\left(n^{-1 / 2+3 \varepsilon}\right),
$$

where $\vec{\alpha}$ denotes the vector composed of the diagonal elements of the matrix $\hat{Q}^{-1}$.

Using the property of the trace function $\operatorname{tr}(X Y)=\operatorname{tr}(Y X)$, we have that

$$
\operatorname{tr}\left(\Phi^{2}\right)=\operatorname{tr}\left(\left(B_{\text {skew }} \hat{Q}^{-1}\right)^{2}\right)+\operatorname{tr}\left(\left(B_{\text {diag }} \hat{Q}^{-1}\right)^{2}\right)+2 \operatorname{tr}\left(B_{\text {skew }} \hat{Q}^{-1} B_{\text {diag }} \hat{Q}^{-1}\right) .
$$

Since $B_{\text {skew }}$ is the skew-symmetric matrix and $\hat{Q}^{-1} B_{\text {diag }} \hat{Q}^{-1}$ is the symmetric matrix

$$
\operatorname{tr}\left(B_{\text {skew }} \hat{Q}^{-1} B_{\text {diag }} \hat{Q}^{-1}\right)=0 \text {. }
$$

One can show that

$$
\begin{aligned}
\operatorname{tr}\left(X^{2}\right) & \leq\|X\|_{H S}^{2}, \\
\|X Y\|_{H S} & \leq\|X\|_{H S}\|Y\|_{2} .
\end{aligned}
$$

Therefore we get that

$$
\left|\operatorname{tr}\left(\left(B_{\text {skew }} \hat{Q}^{-1}\right)^{2}\right)\right| \leq\left\|B_{\text {skew }} \hat{Q}^{-1}\right\|_{H S}^{2}
$$


Since the algebraic connectivity $\lambda_{1} \geq \sigma n$, using (5.9), we obtain that as $n \rightarrow \infty$

$$
\left\|B_{\text {skew }} \hat{Q}^{-1}\right\|_{H S} \leq\left\|\hat{Q}^{-1}\right\|_{2}\left\|B_{\text {skew }}\right\|_{H S} \leq \frac{1}{\lambda_{1}}\left\|B_{\text {skew }}\right\|_{H S}=O\left(n^{-1 / 2+\varepsilon}\right) .
$$

Using (5.9) and (5.15), we get that

$$
\left|\operatorname{tr}\left(\left(B_{\text {diag }}-\Lambda\right) \hat{Q}^{-1} B_{\text {diag }} \hat{Q}^{-1}\right)\right| \leq n \frac{1}{\lambda_{1}^{2}}\left\|\left(B_{\text {diag }}-\Lambda\right)\right\|\left\|_{2}\right\| B_{\text {diag }} \|_{2}=O\left(n^{-1+4 \varepsilon}\right)
$$

and

$$
\left|\operatorname{tr}\left(\left(B_{\text {diag }}-\Lambda\right) \hat{Q}^{-1}\left(B_{\text {diag }}-\Lambda\right) \hat{Q}^{-1}\right)\right| \leq n \frac{1}{\lambda_{1}^{2}}\left\|\left(B_{\text {diag }}-\Lambda\right)\right\|_{2}^{2}=O\left(n^{-2+6 \varepsilon}\right) .
$$

Thus

$$
\operatorname{tr}\left(\left(B_{\text {diag }} \hat{Q}^{-1}\right)^{2}\right)=\operatorname{tr}\left(\left(\Lambda \hat{Q}^{-1}\right)^{2}\right)+O\left(n^{-1+4 \varepsilon}\right) .
$$

Combining (5.18), (5.19), (5.21), (5.22) and (5.25), we obtain that

$$
\operatorname{tr}\left(\Phi^{2}\right)=\operatorname{tr}\left(\left(\Lambda \hat{Q}^{-1}\right)^{2}\right)+O\left(n^{-1+4 \varepsilon}\right) .
$$

Using (5.17) and (5.26) in (5.12), we get that

$$
\operatorname{det}(I+i \Phi)=\exp \left(i \vec{\theta}^{T} Q \vec{\alpha}+\frac{\operatorname{tr}\left(\left(\Lambda \hat{Q}^{-1}\right)^{2}\right)}{2}+O\left(n^{-1 / 2+3 \varepsilon}\right)\right)
$$

Combining (5.7), (5.8) and (5.27), we obtain (5.5).

We denote by $P(\vec{\theta})$ the orthogonal projection onto the space $L$.

Lemma 5.2. Let the assumptions of Theorem 2.1 hold. Let $\hat{Q}=Q+J$, where $J$ denotes the matrix with every entry 1 . For positive constants $a, b, c, d_{1}, d_{2}$ let sequence of vectors $\left\{\vec{\alpha}_{n}\right\}$ and sequence of differentiable functions $\left\{R_{n}(\vec{\theta})\right\}$ be such that

$$
\begin{gathered}
\left\|\vec{\alpha}_{n}\right\|_{\infty} \leq c / n, \\
\left|R_{n}(\vec{\theta})\right| \leq d_{1} \frac{\vec{\theta}^{T} \hat{Q} \vec{\theta}}{n} \\
R_{n}(\vec{\theta})=R_{n}(P(\vec{\theta}))
\end{gathered}
$$

and for $\vec{\theta} \in U_{n}\left(\frac{4}{\sigma} n^{-1 / 2+\varepsilon}\right)$

$$
\left\|\frac{\partial R_{n}(\vec{\theta})}{\partial \vec{\theta}}\right\|_{\infty} \leq d_{2} n^{-1 / 2+\varepsilon}
$$


For $n \geq 2$ define

$$
J_{n}=\int_{L \cap V_{0}} \exp \left(i \overrightarrow{\theta^{T}} Q \overrightarrow{\alpha_{n}}-a \sum_{\left(v_{j}, v_{k}\right) \in E G} \Delta_{j k}^{2}-b \sum_{\left(v_{j}, v_{k}\right) \in E G} \Delta_{j k}^{4}+R_{n}(\vec{\theta})\right) d L .
$$

Then as $n \rightarrow \infty$

$$
J_{n}=\Theta_{k_{1}, k_{2}}\left(\pi^{\frac{n-1}{2}} a^{-\frac{n-1}{2}} n^{1 / 2} / \sqrt{\operatorname{det} \hat{Q}}\right),
$$

where constants $k_{1}, k_{2}>0$ depend only on $a, b, c, d_{1}, d_{2}$ and $\sigma$.

Lemma 5.2 is proved in Section 8.

Lemma 5.3. Let the assumptions of Theorem 2.1 hold. Then as $n \rightarrow \infty$

$$
S_{0}=\Theta_{k_{1}, k_{2}}\left(2^{\frac{n-1}{2}} \pi^{\frac{n+1}{2}} n^{-1} \sqrt{\operatorname{det} \hat{Q}}\right)
$$

where constants $k_{1}, k_{2}>0$ depend only on $\sigma$.

Proof of Lemma 5.3. Using formula (5.2) with $r=1,2 \ldots, n$ and summing, we obtain that

$$
\begin{aligned}
n S_{0} & =\sum_{r=1}^{n} \pi n^{1 / 2} \int_{L \cap V_{0}} \prod_{\left(v_{j}, v_{k}\right) \in E G} \cos \Delta_{j k} \sum_{T \in \mathcal{T}_{r}} \prod_{\left(v_{j}, v_{k}\right) \in E T}\left(1+i \tan \Delta_{j k}\right) d L \\
& =\pi n^{1 / 2} \int_{L \cap V_{0}} \prod_{\left(v_{j}, v_{k}\right) \in E G} \cos \Delta_{j k} \sum_{r=1}^{n} \sum_{T \in \mathcal{T}_{r}} \prod_{\left(v_{j}, v_{k}\right) \in E T}\left(1+i \tan \Delta_{j k}\right) d L .
\end{aligned}
$$

By Taylor's theorem we have that for $\vec{\theta} \in V_{0}$

$$
\prod_{\left(v_{j}, v_{k}\right) \in E G} \cos \Delta_{j k}=\exp \left(-\frac{1}{2} \sum_{\left(v_{j}, v_{k}\right) \in E G} \Delta_{j k}^{2}-\frac{1}{12} \sum_{\left(v_{j}, v_{k}\right) \in E G} \Delta_{j k}^{4}+O\left(n^{-1+6 \varepsilon}\right)\right) .
$$

Combining (5.35) with (5.36) and Lemma 5.1, we obtain that as $n \rightarrow \infty$

$$
S_{0} \sim \pi \frac{\operatorname{det} \hat{Q}}{n^{3 / 2}} \int_{L \cap V_{0}} \exp \left(i \vec{\theta}^{T} Q \vec{\alpha}-\frac{1}{2} \sum_{\left(v_{j}, v_{k}\right) \in E G} \Delta_{j k}^{2}-\frac{1}{12} \sum_{\left(v_{j}, v_{k}\right) \in E G} \Delta_{j k}^{4}+R(\vec{\theta})\right) d L
$$

where $\vec{\alpha}$ denotes the vector composed of the diagonal elements of the matrix $\hat{Q}^{-1}$ and

$$
R(\vec{\theta})=\operatorname{tr}\left(\Lambda \hat{Q}^{-1}\right)^{2}
$$


where $\Lambda$ is the diagonal matrix whose diagonal elements are equal to the components of the vector $Q \vec{\theta}$. Since vector $[1,1 \ldots, 1]^{T}$ is the eigenvector of $Q$, corresponding to eigenvalue 0 , we have that

$$
Q \vec{\theta}=Q P(\vec{\theta})
$$

Thus

$$
R(\vec{\theta})=R(P(\vec{\theta}))
$$

Note that Lemma 3.5 implies as $n \rightarrow \infty$

$$
\|\vec{\alpha}\|_{\infty} \leq c_{1} / n
$$

where $c_{1}=c_{1}(\sigma)>0$. Since the algebraic connectivity $\lambda_{1} \geq \sigma n$, using (5.20), we get that

$$
\begin{aligned}
|R(\vec{\theta})| & \leq\left\|\Lambda \hat{Q}^{-1}\right\|_{H S}^{2} \leq\|\Lambda\|_{H S}^{2}\left\|\hat{Q}^{-1}\right\|_{2}^{2} \\
& =\|Q \vec{\theta}\|_{2}^{2}\left\|\hat{Q}^{-1}\right\|_{2}^{2} \leq\|Q\|_{2}^{2}\left\|\hat{Q}^{-1}\right\|_{2}^{2}\|\vec{\theta}\|_{2}^{2} \\
& \leq \lambda_{n-1}^{2} \frac{1}{\lambda_{1}^{2}}\|\vec{\theta}\|_{2}^{2} \leq n^{2} \frac{1}{\lambda_{1}^{2}} \frac{\vec{\theta}^{T} \hat{Q} \vec{\theta}}{\lambda_{1}} \leq \frac{1}{\sigma^{3}} \frac{\vec{\theta}^{T} \hat{Q} \vec{\theta}}{n} .
\end{aligned}
$$

Note that for $\vec{\theta} \in U_{n}\left(\frac{4}{\sigma} n^{-1 / 2+\varepsilon}\right)$ and for some $1 \leq k \leq n$

$$
\|\Lambda\|_{2} \leq \sum_{j=1}^{n}\left|\Delta_{j k}\right|=O\left(n^{1 / 2+\varepsilon}\right) .
$$

For $1 \leq k \leq n$ we denote by $\left(\hat{Q}^{-1}\right)_{k}$ the $k$-th column of the matrix $\hat{Q}^{-1}$. Using again $\lambda_{1} \geq \sigma n$, we get that

$$
1=\left\|\hat{Q}\left(\hat{Q}^{-1}\right)_{k}\right\|_{2} \geq \lambda_{1}\left\|\left(\hat{Q}^{-1}\right)_{k}\right\|_{2} \geq \sigma n\left\|\left(\hat{Q}^{-1}\right)_{k}\right\|_{2} .
$$

Note that

$$
\frac{\partial R}{\partial \theta_{k}}=2 \operatorname{tr}\left(\frac{\partial \Lambda}{\partial \theta_{k}} \hat{Q}^{-1} \Lambda \hat{Q}^{-1}\right)=2 d_{k}\left(\hat{Q}^{-1} \Lambda \hat{Q}^{-1}\right)_{k k}+2 \operatorname{tr}\left(\tilde{\Lambda} \hat{Q}^{-1} \Lambda \hat{Q}^{-1}\right),
$$

where $\left(\hat{Q}^{-1} \Lambda \hat{Q}^{-1}\right)_{k k}$ denotes $(k, k)$-th element of the matrix $\hat{Q}^{-1} \Lambda \hat{Q}^{-1}$ and the matrix $\tilde{\Lambda}$ is such that for any $1 \leq j \leq n$ the diagonal element $\left|\Lambda_{j j}\right| \leq 1$. Since the algebraic connectivity $\lambda_{1} \geq \sigma n$, using (5.43) (5.44), we get that as $n \rightarrow \infty$

$$
\left|\operatorname{tr}\left(\tilde{\Lambda} \hat{Q}^{-1} \Lambda \hat{Q}^{-1}\right)\right| \leq n\left\|\tilde{\Lambda} \hat{Q}^{-1} \Lambda \hat{Q}^{-1}\right\|_{2} \leq n\|\tilde{\Lambda}\|_{2}\|\Lambda\|_{2} \frac{1}{\lambda_{1}^{2}}=O\left(n^{-1 / 2+\varepsilon}\right)
$$

and

$$
2 d_{k}\left(\hat{Q}^{-1} \Lambda \hat{Q}^{-1}\right)_{k k}=2 d_{k} \Lambda_{k k}\left\|\left(\hat{Q}^{-1}\right)_{k}\right\|_{2}^{2}=O\left(n^{-1 / 2+\varepsilon}\right) .
$$

Thus for some $d>0$, depending only on $\sigma$

$$
\left\|\frac{\partial R_{n}(\vec{\theta})}{\partial \vec{\theta}}\right\|_{\infty} \leq d n^{-1 / 2+\varepsilon} .
$$

Combining (5.37), (5.40), (5.41), (5.42), (5.48) and using Lemma 5.2 we obtain (5.34). 


\section{The insignificant parts of the integral}

In this section we prove that $S_{0}$ contributes almost all of $S$, even though it involves only a tiny part of the region of integration, compare with Section 4 of [6]. We continue to use the same value of $\varepsilon$ as in the previous section.

Let assumptions of Theorem 2.1 hold. Define $E^{\prime} T=\left\{\left(v_{j}, v_{k}\right),\left(v_{k}, v_{j}\right) \mid\left(v_{j}, v_{k}\right) \in E T\right\}$. We express the integrand of (4.7) as

$$
F(\vec{\theta})=\sum_{T \in T_{r}} \prod_{j} f_{k \in E G}(T, \vec{\theta})
$$

where

$$
f_{j k}(T, \vec{\theta})= \begin{cases}\cos \Delta_{j k}\left(1+i \tan \Delta_{j k}\right), & \left(v_{j}, v_{k}\right) \in E T \\ \cos \Delta_{j k}\left(1-i \tan \Delta_{j k}\right), & \left(v_{k}, v_{j}\right) \in E T \\ \cos \Delta_{j k}, & \text { otherwise }\end{cases}
$$

Note that $\left|f_{j k}(T, \vec{\theta})\right| \leq 1$ for all values of the parameters. One can show that

$$
|\cos (x)| \leq \exp \left(-\frac{1}{2} x^{2}\right) \text { for }|x| \leq \frac{9}{16} \pi
$$

Divide the interval $\left[-\frac{1}{2} \pi, \frac{1}{2} \pi\right] \bmod \pi$ into 32 equal intervals $H_{0}, \ldots, H_{31}$ such that $H_{0}=\left[-\frac{1}{64} \pi, \frac{1}{64} \pi\right]$. For each $j$, define the region $W_{j} \subseteq U_{n}(\pi / 2)$ as the set of points having at least $\frac{1}{32} n$ coordinates in $H_{j}$. Clearly, the $W_{j}$ 's cover $U_{n}(\pi / 2)$ and also each $W_{j}$ can be mapped to $W_{0}$ by a uniform translation of the $\theta_{j} \bmod \pi$. This mapping preserves the integrand of (4.7) and also maps $V_{0}$ to itself, so we have that

$$
\int_{U_{n}(\pi / 2)-V_{0}}|F(\theta)| d \vec{\theta} \leq 32 Z
$$

where

$$
Z=\int_{W_{0}-V_{0}}|F(\theta)| d \vec{\theta}
$$

We proceed by defining integrals $S_{1}, \ldots, S_{4}$ in such a way that $Z$ is obviously bounded by their sum. We then show that $S_{j}=o\left(S_{0}\right)$ for $j=1,2,3,4$ separately. Write

$$
F(\vec{\theta})=F_{a}(\vec{\theta})+F_{b}(\vec{\theta})
$$

where $F_{a}(\theta)$ and $F_{b}(\theta)$ are defined by restricting the sum to trees with maximum degree greater than $\sigma n / 4$ and no more than $\sigma n / 4$, respectively. Also define regions $V_{1}$ and $V_{2}$ as follows.

$$
\begin{aligned}
& V_{1}=\left\{\vec{\theta} \in W_{0}|| \theta_{j} \mid \geq \frac{1}{32} \pi \text { for fewer than } n^{\varepsilon} \text { values of } j\right\} \\
& V_{2}=\left\{\vec{\theta} \in V_{1}|| \theta_{j} \mid \geq \frac{1}{16} \pi \text { for at least one value of } j\right\}
\end{aligned}
$$


Then our four integrals can be defined as

$$
\begin{aligned}
& S_{1}=\int_{W_{0}-V_{1}}|F(\vec{\theta})| d \vec{\theta}, \\
& S_{2}=\int_{V_{1}}\left|F_{a}(\vec{\theta})\right| d \vec{\theta}, \\
& S_{3}=\int_{V_{2}}\left|F_{b}(\vec{\theta})\right| d \vec{\theta}, \\
& S_{4}=\int_{V_{1}-V_{2}-V_{0}}\left|F_{b}(\vec{\theta})\right| d \vec{\theta} .
\end{aligned}
$$

We begin with $S_{1}$. Let $h$ be the function from Lemma 3.9 for the set $A=\left\{v_{j}|| \theta_{j} \mid \leq\right.$ $\left.\frac{1}{64} \pi\right\}$. We denote $l_{\text {min }}$ such natural number that inequality

$$
\left|\theta_{j}\right| \geq \frac{1}{64} \pi(1+l / H)
$$

holds for at least $n^{\varepsilon} / H$ indices of the set $\left\{j \mid h\left(v_{j}\right)=l\right\}$. Existence of $l_{\text {min }}$ follows from the definition of the region $V_{1}$. If $\theta_{j}$ and $\theta_{k}$ are such that

$$
\left|\theta_{j}\right| \geq \frac{1}{64} \pi\left(1+l_{\text {min }} / H\right) \text { and }\left|\theta_{k}\right| \leq \frac{1}{64} \pi\left(1+\left(l_{\text {min }}-1\right) / H\right)
$$

or vice versa, but $\left(v_{j}, v_{k}\right) \notin E^{\prime} T$, we have that $\left|f_{j k}(T, \vec{\theta})\right| \leq \cos \left(\frac{1}{64} \pi / H\right)$. This includes at least $\left(\alpha n-n^{\varepsilon}\right) \frac{n^{\varepsilon}}{H}-n$ edges $\left(v_{j}, v_{k}\right) \in E G$. Using (3.1) and (3.33), we get that as $n \rightarrow \infty$

$$
S_{1} \leq t(G) \pi^{n}\left(\cos \frac{\pi}{64 H}\right)^{\left(\alpha n-n^{\varepsilon}\right) \frac{n^{\varepsilon}}{H}-n}=O\left(\exp \left(-c n^{1+\varepsilon}\right)\right) 2^{\frac{n-1}{2}} \pi^{\frac{n+1}{2}} n^{-1} \sqrt{\operatorname{det} \hat{Q}}
$$

for some constant $c>0$ depending only on $\sigma$.

To bound $S_{2}$, we first note from Lemma 3.7 that the number of trees with maximum de- gree greater than $\sigma n / 4$ is less than $c_{3}^{n} \operatorname{det} \hat{Q} /(\sigma n / 4)$ !. Using (6.3), we see that

$$
\left|f_{j k}(T, \vec{\theta})\right| \leq \exp \left(-\frac{1}{2} \Delta_{j k}^{2}\right)
$$

except for at most $n^{2 \varepsilon}$ pairs $(j, k)$ with $\left|\Delta_{j k}\right| \geq \frac{1}{16} \pi$ and fewer than $n$ pairs in $E^{\prime} T$. In those excluded cases the value $\exp \left(-\frac{1}{2} \Delta_{j k}^{2}\right)$ may be high by a factor $\exp \left(\frac{1}{2} \pi^{2}\right)$. Hence, we have that

$$
S_{2} \leq \frac{c_{3}^{n} \operatorname{det} \hat{Q}}{(\sigma n / 4) !} \exp \left(\frac{1}{2} \pi^{2}\left(n+n^{2 \varepsilon}\right)\right) \int_{U_{n}(\pi / 2)} \exp \left(-\frac{1}{2} \sum_{\left(v_{j}, v_{k}\right) \in E G} \Delta_{j k}^{2}\right) d \vec{\theta} .
$$


Lemma 6.1. Let the assumptions of Lemma 3.2 hold. Then

$$
\int_{U_{n}(\pi / 2)} \exp \left(-\frac{1}{2} \sum_{\left(v_{j}, v_{k}\right) \in E G} \Delta_{j k}^{2}\right) d \vec{\theta} \leq \frac{2^{\frac{n-1}{2}} \pi^{\frac{n+1}{2}} n}{\sqrt{\operatorname{det} \hat{Q}}} .
$$

Lemma 6.1 is proved in Section 8. Combining (6.13) and (6.14), we obtain that as $n \rightarrow \infty$

$$
S_{2}=O\left(n^{-c n}\right) 2^{\frac{n-1}{2}} \pi^{\frac{n+1}{2}} n^{-1} \sqrt{\operatorname{det} \hat{Q}}
$$

for some constant $c>0$ depending only on $\sigma$.

We denote by $G_{T}$ the graph which arises from $G$ by removing all edges of the tree $T$. Let $G_{T, \vec{\theta}}$ be the graph resulting from from $G_{T}$ by removing vertices, corresponding to those values of $j$ for which $\left|\theta_{j}\right| \geq \frac{1}{16} \pi$.

For $1 \leq r \leq n^{\varepsilon}$ let $S_{3}(r)$ denote the contribution to $S_{3}$ of those $\theta \in V_{2}$ such that $\left|\theta_{j}\right| \geq \frac{1}{16} \pi$ for exactly $r$ values of $j$. If $\left|\theta_{j}\right| \leq \frac{1}{32} \pi$ and $\left|\theta_{k}\right| \geq \frac{1}{16} \pi$ or vice versa, we have that

$$
\left|f_{j k}(T, \vec{\theta})\right| \leq \cos \left(\frac{1}{32} \pi\right)
$$

unless $\left(v_{j}, v_{k}\right) \in E^{\prime} T$. This includes at least $r\left(\sigma n / 2-\sigma n / 4-n^{\varepsilon}\right)$ pairs $(j, k)$, because the degree of any vertex of the graph $G$ is at least $\sigma n / 2$, see (3.2). For pairs $(j, k)$ such that $\left|\theta_{j}\right|,\left|\theta_{k}\right| \leq \frac{1}{16} \pi$, but $\left(v_{j}, v_{k}\right) \notin E^{\prime} T$, we use (6.12). We put $\overrightarrow{\theta^{\prime}}=\left(\theta_{1}, \ldots, \theta_{n-r}\right)$. Then,

$$
S_{3}(r) \leq \pi^{r}\left(\cos \frac{\pi}{32}\right)^{r\left(\sigma n / 4-n^{\varepsilon}\right)} \sum_{(r)} \sum_{T} \int_{U_{n-r}(\pi / 2)} \exp \left(-\frac{1}{2} \sum_{\left(v_{j}, v_{k}\right) \in E G_{T, \vec{\theta}}} \Delta_{j k}^{2}\right) d{\overrightarrow{\theta^{\prime}}}^{\prime}
$$

where the first sum is over choices of those values of $j$ for which $\left|\theta_{j}\right| \geq \frac{1}{16} \pi$ and the second sum is over trees with maximum degree $\sigma n / 4$. Using Lemma 3.8 and then Lemma 3.1 and Lemma 3.6 for the graph $G_{T}$, we obtain that

$$
\lambda_{1}\left(G_{T, \vec{\theta}}\right) \geq \sigma n / 2-n^{\varepsilon}
$$

and

$$
\operatorname{det} \hat{Q}\left(G_{T, \vec{\theta}}\right) \geq \frac{\operatorname{det} \hat{Q}}{\left(c_{5} n\right)^{r}}
$$

where $c_{5}=c_{5}(\sigma)>0$ and $\hat{Q}=\hat{Q}(G)$. According to Lemma 6.1, we have that

$$
\int_{U_{n-r}(\pi / 2)} \exp \left(-\frac{1}{2} \sum_{\left(v_{j}, v_{k}\right) \in E G_{T, \vec{\theta}}} \Delta_{j k}^{2}\right) d \overrightarrow{\theta^{\prime}} \leq \frac{2^{\frac{n-r-1}{2} \pi^{\frac{n-r+1}{2}} n}}{\sqrt{\operatorname{det} \hat{Q}\left(G_{T, \vec{\theta}}\right)}} .
$$

Combining (6.17) with (6.19), (6.20) and allowing $n^{r}$ for the choice of those values of $j$ for which $\left|\theta_{j}\right| \geq \frac{1}{16} \pi$, we obtain that

$$
S_{3}(r) \leq 2^{\frac{n-r-1}{2}} \pi^{\frac{n+r+1}{2}} n^{r+1}\left(\cos \frac{\pi}{32}\right)^{r\left(\sigma n / 4-n^{\varepsilon}\right)} \frac{t(G)\left(c_{5} n\right)^{r / 2}}{\sqrt{\operatorname{det} \hat{Q}}}
$$


and, using (3.33), we can calculate that

$$
S_{3}=\sum_{r=1}^{n^{\varepsilon}} S_{3}(r)=O\left(c^{-n}\right) 2^{\frac{n-1}{2}} \pi^{\frac{n+1}{2}} n^{-1} \sqrt{\operatorname{det} \hat{Q}}
$$

for some constant $c>1$ depending only on $\sigma$.

Since $\Delta_{j k} \leq \frac{1}{8} \pi$ for $\vec{\theta} \in V_{1}-V_{2}-V_{0}$ and the integrand is invariant under uniform translation of all the $\theta_{j}$ 's $\bmod \pi$, we can fix $\bar{\theta}=0$ and multiply it by the ratio of its range $\pi$ to the length $n^{-1 / 2}$ of the vector $\frac{1}{n}[1,1, \ldots, 1]^{T}$. Thus we get that

$$
S_{4} \leq \pi n^{1 / 2} \int_{L \cap U_{n}(\pi / 8)-V_{0}}\left|F_{b}(\vec{\theta})\right| d L
$$

where $L$ denotes the orthogonal complement to the vector $[1,1, \ldots, 1]^{T}$. In a similar way as $(6.17)$ we find that

$$
S_{4} \leq \pi n^{1 / 2} \sum_{T} \int_{L \cap U_{n}(\pi / 8)-V_{0}} \exp \left(-\frac{1}{2} \sum_{\left(v_{j}, v_{k}\right) \in E G_{T}} \Delta_{j k}^{2}\right) d L
$$

where the first sum is over trees with maximum degree $\sigma n / 4$.

Lemma 6.2. Let the assumptions of Lemma 3.2 hold. Then as $n \rightarrow \infty$

$$
\int_{L-U_{n}\left(n^{-1 / 2+\varepsilon}\right)} \exp \left(-\frac{1}{2} \sum_{\left(v_{j}, v_{k}\right) \in E G} \Delta_{j k}^{2}\right) d L=O\left(\exp \left(-c n^{2 \varepsilon}\right)\right) \frac{2^{\frac{n-1}{2}} \pi^{\frac{n-1}{2}} n^{1 / 2}}{\sqrt{\operatorname{det} \hat{Q}}}
$$

for some $c>0$ depending only on $\sigma$.

Lemma 6.2 is proved in Section 8. Using Lemma 3.8 and combining (6.25), (6.24) and (3.33), we obtain that as $n \rightarrow \infty$

$$
S_{4}=O\left(\exp \left(-c n^{2 \varepsilon}\right)\right) t(G) \frac{2^{\frac{n-1}{2}} \pi^{\frac{n+1}{2}} n}{\sqrt{\operatorname{det} \hat{Q}}}=O\left(\exp \left(-c n^{2 \varepsilon}\right)\right) 2^{\frac{n-1}{2}} \pi^{\frac{n+1}{2}} n^{-1} \sqrt{\operatorname{det} \hat{Q}}
$$

for some $c>0$ depending only on $\sigma$. Combining (6.11), (6.15), (6.22), (6.26) and Lemma 5.3 , we obtain the desired result.

Lemma 6.3. Let the assumptions of Theorem 2.1 hold. Then as $n \rightarrow \infty$

$$
S=\left(1+O\left(\exp \left(-c n^{2 \varepsilon}\right)\right)\right) S_{0}
$$

for some $c>0$ depending only on $\sigma$. 


\section{$7 \quad$ Proof of Lemma 3.2}

According to (4.6) and (4.7)

$$
\operatorname{Eul}(G)=\prod_{j=1}^{n}\left(\frac{d_{j}}{2}-1\right) ! 2^{|E G|-n+1} \pi^{-n} S
$$

where

$$
S=\int_{U_{n}(\pi / 2)} \prod_{\left(v_{j}, v_{k}\right) \in E G} \cos \Delta_{j k} \sum_{T \in \mathcal{T}_{r}} \prod_{\left(v_{j}, v_{k}\right) \in E T}\left(1+i \tan \Delta_{j k}\right) d \vec{\theta} .
$$

Combining Lemma 5.3 and Lemma 6.3 we get that as $n \rightarrow \infty$

$$
S=\Theta_{k_{1}, k_{2}}\left(2^{\frac{n-1}{2}} \pi^{\frac{n+1}{2}} n^{-1} \sqrt{\operatorname{det} \hat{Q}}\right)
$$

where constants $k_{1}, k_{2}>0$ depend only on $\sigma$. Taking into account (3.33) we obtain (2.11).

If for some $\sigma>1 / 2$ the degree of each vertex of the graph $G$ at least $\sigma n$, we can use (3.3) and get that

$$
\lambda_{1}(G) \geq 2 \min _{j} d_{j}-n+2>(2 \sigma-1) n .
$$

\section{Proofs of Lemma 5.2, Lemma 6.1 and Lemma 6.2}

Let assumptions of Lemma 3.2 hold. We define

$$
\vec{\phi}=\vec{\phi}(\vec{\theta})=\left(\phi_{1}(\vec{\theta}), \ldots \phi_{n}(\vec{\theta})\right)=\hat{Q} \vec{\theta}
$$

We continue to use notation $P(\vec{\theta})$ for the orthogonal projection onto the space $L$, where $L$ is the orthogonal complement to the vector $[1,1, \ldots, 1]^{T}$. For any $a>0$ we have that

$$
\int_{\mathbb{R}^{n}} e^{-a \vec{\theta}^{T} \hat{Q} \vec{\theta}} d \vec{\theta}=\pi^{n / 2} a^{-n / 2} / \sqrt{\operatorname{det} \hat{Q}}
$$

and

$$
\int_{L} e^{-a \vec{\theta}^{T} \hat{Q} \vec{\theta}} d L=\int_{L} e^{-a \vec{\theta}^{T} Q \vec{\theta}} d L=\pi^{\frac{n-1}{2}} a^{-\frac{n-1}{2}} n^{1 / 2} / \sqrt{\operatorname{det} \hat{Q}} .
$$

Proof of Lemma 6.1. Note that

$$
\sum_{\left(v_{j}, v_{k}\right) \in E G} \Delta_{j k}^{2}=\vec{\theta}^{T} Q \vec{\theta}
$$


Since diagonal of $U_{n}(\pi / 2)$ is equal to $\pi n^{1 / 2}$ and $Q \vec{\theta}=Q P(\vec{\theta})$ we have that

$$
\int_{U_{n}(\pi / 2)} \exp \left(-\frac{1}{2} \sum_{\left(v_{j}, v_{k}\right) \in E G} \Delta_{j k}^{2}\right) d \vec{\theta} \leq \pi n^{1 / 2} \int_{L} e^{-\frac{1}{2} \vec{\theta}^{T} Q \vec{\theta}} d L .
$$

Using (8.3), we obtain (6.14).

Note that for some $g_{1}(\vec{\theta})=g_{1}\left(\theta_{2}, \ldots, \theta_{n}\right)$

$$
\vec{\theta}^{T} \hat{Q} \vec{\theta}=\frac{\phi_{1}(\vec{\theta})^{2}}{d_{1}+1}+g_{1}(\vec{\theta})
$$

Using (3.9), we get that as $n \rightarrow \infty$

$$
\begin{aligned}
\int_{\mathbb{R}^{n}} e^{-a \vec{\theta}^{T} \hat{Q} \vec{\theta}} d \vec{\theta} & =\int_{-\infty}^{+\infty} \cdots \int_{-\infty}^{+\infty} e^{-a g_{1}\left(\theta_{2}, \ldots, \theta_{n}\right)}\left(\int_{-\infty}^{+\infty} e^{-a \frac{\phi_{1}(\vec{\theta})^{2}}{d_{1}+1}} d \theta_{1}\right) d \theta_{2} \ldots d \theta_{n} \\
& =\left(1+O\left(\exp \left(-\tilde{c} n^{2 \varepsilon}\right)\right)\right) \int_{\left|\phi_{1}(\vec{\theta})\right| \leq \frac{1}{2} c_{\infty}^{-1} n^{1 / 2+\varepsilon}} e^{-a \vec{\theta}^{T} \hat{Q} \vec{\theta}} d \vec{\theta}
\end{aligned}
$$

for some $\tilde{c}>0$ depending only on $\sigma$ and $a$, where $c_{\infty}$ is the constant of Lemma 3.2. Combining similar expressions for $\phi_{1}, \phi_{2}, \ldots \phi_{n}$, we obtain that as $n \rightarrow \infty$

$$
\int_{\|\vec{\phi}(\vec{\theta})\|_{\infty} \leq \frac{1}{2} c_{\infty}^{-1} n^{1 / 2+\varepsilon}} e^{-a \vec{\theta}^{T} \hat{Q} \vec{\theta}} d \vec{\theta}=\left(1+O\left(\exp \left(-c n^{2 \varepsilon}\right)\right)\right) \int_{\mathbb{R}^{n}} e^{-a \vec{\theta}^{T} \hat{Q} \vec{\theta}} d \vec{\theta}
$$

for some $c>0$ depending only on $\sigma$ and $a$. Using Lemma 3.2, we get that as $n \rightarrow \infty$

$$
\int_{U_{n}\left(\frac{1}{2} n^{-1 / 2+\varepsilon}\right)} e^{-a \vec{\theta}^{T} \hat{Q} \vec{\theta}} d \vec{\theta}=\left(1+O\left(\exp \left(-c n^{2 \varepsilon}\right)\right)\right) \int_{\mathbb{R}^{n}} e^{-a \vec{\theta}^{T} \hat{Q} \vec{\theta}} d \vec{\theta} .
$$

Proof of Lemma 6.2. Note that

$$
\|P(\vec{\theta})\|_{\infty}=\left\|\vec{\theta}-\bar{\theta}[1,1, \ldots, 1]^{T}\right\|_{\infty} \leq 2\|\vec{\theta}\|_{\infty}
$$

where

$$
\bar{\theta}=\frac{\theta_{1}+\theta_{2}+\ldots \theta_{n}}{n}
$$

Thus

$$
U_{n}\left(\frac{1}{2} n^{-1 / 2+\varepsilon}\right) \subset\left\{\vec{\theta} \mid P(\vec{\theta}) \in U_{n}\left(n^{-1 / 2+\varepsilon}\right)\right\}
$$


Since $Q \vec{\theta}=Q P(\vec{\theta})$, using (8.4) and (8.12), we get that

$$
\begin{aligned}
& \int_{L \cap U_{n}\left(n^{-1 / 2+\varepsilon}\right)} \exp \left(-\frac{1}{2} \sum_{\left(v_{j}, v_{k}\right) \in E G} \Delta_{j k}^{2}\right) d L=\int_{L \cap U_{n}\left(n^{-1 / 2+\varepsilon}\right)} e^{-\frac{1}{2} \vec{\theta}^{T} Q \vec{\theta}} d L \\
= & \int_{P(\vec{\theta}) \in U_{n}\left(n^{-1 / 2+\varepsilon}\right)} e^{-\frac{1}{2} \vec{\theta}^{T} \hat{Q} \vec{\theta}} d \vec{\theta} / \int_{-\infty}^{+\infty} e^{-\frac{1}{2} n x^{2}} d x \geq \frac{n^{1 / 2}}{\sqrt{2 \pi}} \int_{U_{n}\left(\frac{1}{2} n^{-1 / 2+\varepsilon}\right)} e^{-\frac{1}{2} \vec{\theta}^{T} \hat{Q} \vec{\theta}} d \vec{\theta} .
\end{aligned}
$$

Combining (8.2), (8.9) and (8.13) we obtain (6.25).

To prove Lemma 5.2 we separate the integrand in (5.32) into three factors.

- $\exp \left(i \overrightarrow{\theta^{T}} Q \overrightarrow{\alpha_{n}}\right)$ — the oscillatory factor,

- $\exp \left(a \sum_{\left(v_{j}, v_{k}\right) \in E G} \Delta_{j k}^{2}\right)$ - the regular factor,

- $\exp \left(b \sum_{\left(v_{j}, v_{k}\right) \in E G} \Delta_{j k}^{4}+R_{n}(\vec{\theta})\right)$ - the residual factor.

The proof consists of the following steps.

1. In Lemma 8.3 we estimate an integral analogous to (5.32) but without an oscillatory factor.

2. Using Lemma 8.5, we get rid of the oscillatory factor in (5.32).

3. Combining Lemma 8.5 and Lemma 8.3, we complete the proof of Lemma 5.2.

At first, we prove two technical statements.

Lemma 8.1. For any $a>0$ and sequence of functions $r_{n}(x)$ such that as $n \rightarrow \infty$

$$
\sup _{|x| \leq n^{-1 / 2+\varepsilon}}\left|r_{n}(x)\right|=o(1)
$$

Then as $n \rightarrow \infty$

$$
\int_{-n^{-1 / 2+\varepsilon}}^{n^{-1 / 2+\varepsilon}} n x^{2} e^{-a n x^{2}+r_{n}(x)} d x=\left(\frac{1}{2 a}+o(1)\right) \int_{-n^{-1 / 2+\varepsilon}}^{n^{-1 / 2+\varepsilon}} e^{-a n x^{2}+r_{n}(x)} d x .
$$

and

$$
\int_{-n^{-1 / 2+\varepsilon}}^{n^{-1 / 2+\varepsilon}} n^{2} x^{4} e^{-a n x^{2}+r_{n}(x)} d x=\left(\frac{3}{4 a^{2}}+o(1)\right) \int_{-n^{-1 / 2+\varepsilon}}^{n^{-1 / 2+\varepsilon}} e^{-a n x^{2}+r_{n}(x)} d x
$$


Proof of Lemma 8.1. Note that

$$
\int_{-n^{\varepsilon}}^{n^{\varepsilon}} t^{2} e^{-a t^{2}} d t=\left(1+O\left(\exp \left(-c n^{2 \varepsilon}\right)\right)\right) \int_{-\infty}^{+\infty} t^{2} e^{-a t^{2}} d t=\left(\frac{1}{2 a}+o(1)\right) \int_{-n^{\varepsilon}}^{n^{\varepsilon}} e^{-a t^{2}} d t
$$

and

$$
\int_{-n^{\varepsilon}}^{n^{\varepsilon}} t^{4} e^{-a t^{2}} d t=\left(1+O\left(\exp \left(-c n^{2 \varepsilon}\right)\right)\right) \int_{-\infty}^{+\infty} t^{4} e^{-a t^{2}} d t=\left(\frac{3}{4 a^{2}}+o(1)\right) \int_{-n^{\varepsilon}}^{n^{\varepsilon}} e^{-a t^{2}} d t .
$$

Using (8.14), we get that as $n \rightarrow \infty$

$$
\sup _{|x| \leq n^{-1 / 2+\varepsilon}}\left|e^{r_{n}(x)}-1\right|=o(1) .
$$

Making the substitution $t=\sqrt{n} x$ and combining (8.17) and (8.18) with (8.19), we obtain (8.15) and (8.16), respectively.

Lemma 8.2. Under the assumptions of Lemma 8.1, let $r_{n}(x)$ be differentiable and as $n \rightarrow \infty$

$$
\sup _{|x| \leq n^{-1 / 2+\varepsilon}}\left|r_{n}^{\prime}(x)\right|=O\left(n^{-1 / 2+3 \varepsilon}\right) .
$$

Then as $n \rightarrow \infty$

$$
\int_{-n^{-1 / 2+\varepsilon}}^{n^{-1 / 2+\varepsilon}} x e^{-a n x^{2}+r_{n}(x)} d x=O\left(n^{-3 / 2+4 \varepsilon}\right) \int_{-n^{-1 / 2+\varepsilon}}^{n^{-1 / 2+\varepsilon}} e^{-a n x^{2}+r_{n}(x)} d x .
$$

Proof of Lemma 8.2. Note that

$$
\int_{0}^{n^{\varepsilon}} t e^{-a t^{2}} d t=\left(1+O\left(\exp \left(-c n^{2 \varepsilon}\right)\right)\right) \int_{0}^{+\infty} t e^{-a t^{2}} d t=\frac{1}{2 a}+O\left(\exp \left(-c n^{2 \varepsilon}\right)\right) .
$$

According to the Mean Value Theorem, we have that for some $|\tilde{x}| \leq|x|$

$$
\left|e^{r_{n}(x)}-e^{r_{n}(-x)}\right|=\left|e^{r_{n}(\tilde{x})} r_{n}^{\prime}(\tilde{x}) 2 x\right| .
$$

Using (8.20), we get that as $n \rightarrow \infty$

$$
\sup _{|x| \leq n^{-1 / 2+\varepsilon}}\left|e^{r_{n}(x)}-e^{r_{n}(-x)}\right|=O\left(n^{-1+4 \varepsilon}\right) .
$$

We have that

$$
\int_{-n^{-1 / 2+\varepsilon}}^{n^{-1 / 2+\varepsilon}} x e^{-a n x^{2}+r_{n}(x)} d x=\int_{0}^{n^{-1 / 2+\varepsilon}} x e^{-a n x^{2}}\left(e^{r_{n}(x)}-e^{r_{n}(-x)}\right) d x .
$$


Making the substitution $t=\sqrt{n} x$ and combining (8.22) with (8.24), we obtain (8.21).

We use notation

$$
\mu_{m}=\sum_{j=1}^{n}\left|\phi_{j}\right|^{m}
$$

According to the Generalized Mean Inequality, we have that

$$
\mu_{1} / n \leq\left(\mu_{4} / n\right)^{1 / 4}
$$

Since

$$
\phi_{k}=\left(d_{k}+1\right) \theta_{k}+\sum_{\left(v_{k}, v_{j}\right) \notin E G} \theta_{j}
$$

and (see (3.18))

$$
d_{k} \geq \sigma n / 2
$$

we obtain that

$$
\left|\theta_{k}\right| \leq \frac{2}{\sigma n}\left(\left|\phi_{k}\right|+\sum_{j \neq k}\left|\theta_{j}\right|\right)
$$

Using Lemma 3.2, we find that

$$
\sum_{j \neq k}\left|\theta_{j}\right| \leq\|\theta\|_{1} \leq \frac{c_{\infty}}{n}\|\phi\|_{1}=\frac{c_{\infty}}{n} \mu_{1}
$$

Combining (8.27), (8.30) and (8.31), we get that

$$
\theta_{k}^{4} \leq \frac{16}{\sigma^{4} n^{4}}\left(\left|\phi_{k}\right|+\frac{c_{\infty}}{n} \mu_{1}\right)^{4} \leq \frac{16}{\sigma^{4} n^{4}}\left(\left|\phi_{k}\right|+c_{\infty}\left(\mu_{4} / n\right)^{1 / 4}\right)^{4} .
$$

Using the inequality $(x+y)^{4} \leq 8\left(x^{4}+y^{4}\right)$, we obtain that

$$
\theta_{k}^{4} \leq c_{\phi} \frac{\phi_{k}^{4}}{n^{4}}+c_{\mu} \frac{\mu_{4}}{n^{5}}
$$

where constants $c_{\phi}, c_{\mu}>0$ depend only on $\sigma$.

Lemma 8.3. Let assumptions of Lemma 3.2 hold. Let $\left\{a_{n}\right\}$ be sequence of positive numbers having limit $a>0$. Then for any $b>0$ as $n \rightarrow \infty$

$$
\int_{U_{n}\left(n^{-1 / 2+\varepsilon)}\right.} \exp \left(-a_{n} \vec{\theta}^{T} \hat{Q} \vec{\theta}-b \sum_{\left(v_{j}, v_{k}\right) \in E G} \Delta_{j k}^{4}\right) d \vec{\theta}=\Theta_{k_{1}, k_{2}}\left(\int_{\mathbb{R}^{n}} e^{-a_{n} \vec{\theta}^{T} \hat{Q} \vec{\theta}} d \vec{\theta}\right),
$$

where constants $k_{1}, k_{2}>0$ depend only on $a, b$ and $\sigma$. 
Proof of Lemma 8.3. Using the inequality $(x+y)^{4} \leq 8\left(x^{4}+y^{4}\right)$, we find that

$$
\sum_{\left(v_{j}, v_{k}\right) \in E G} \Delta_{j k}^{4} \leq 8 n \sum_{j=1}^{n} \theta_{j}^{4}
$$

We define $R_{1}(\vec{\theta})=8 n \sum_{j=1}^{n} \theta_{j}^{4}$. Thus we have that

$$
\int_{U_{n}\left(n^{-1 / 2+\varepsilon}\right)} \exp \left(-a_{n} \vec{\theta}^{T} \hat{Q} \vec{\theta}-b \sum_{\left(v_{j}, v_{k}\right) \in E G} \Delta_{j k}^{4}\right) d \vec{\theta} \geq \int_{\mathbb{R}^{n}} e^{-a_{n} \vec{\theta}^{T} \hat{Q} \vec{\theta}-R_{1}(\vec{\theta})} d \vec{\theta}
$$

Using (8.9), we find that as $n \rightarrow \infty$

$$
\begin{aligned}
\int_{U_{n}\left(n^{-1 / 2+\varepsilon}\right)} \phi_{1}^{4} e^{-a_{n} \vec{\theta}^{T} \hat{Q} \vec{\theta}-R_{1}(\vec{\theta})} d \vec{\theta}= & \int_{U_{n}\left(\frac{4}{\sigma} n^{-1 / 2+\varepsilon}\right)} \phi_{1}^{4} e^{-a_{n} \vec{\theta}^{T} \hat{Q} \vec{\theta}-R_{1}(\vec{\theta})} d \vec{\theta} \\
& +O\left(\exp \left(-c n^{2 \varepsilon}\right)\right) \int_{\mathbb{R}^{n}} e^{-a_{n} \vec{\theta}^{T} \hat{Q} \vec{\theta}} d \vec{\theta}
\end{aligned}
$$

for some $c>0$ depending only on $a$ and $\sigma$. It follows that

$$
\begin{aligned}
\int_{-n^{-1 / 2+\varepsilon}}^{n^{-1 / 2+\varepsilon}} & \cdots \int_{-n^{-1 / 2+\varepsilon}}^{n^{-1 / 2+\varepsilon}}\left(\int_{-\frac{4}{\sigma} n^{-1 / 2+\varepsilon}} \phi_{1}^{4} e^{-a_{n} \overrightarrow{\theta^{T}} \hat{Q} \vec{\theta}-R_{1}(\vec{\theta})} d \theta_{1}\right) d \theta_{2} \ldots d \theta_{n} \\
& =\int_{U_{n}\left(n^{-1 / 2+\varepsilon}\right)} \phi_{1}^{4} e^{-a_{n} \vec{\theta}^{T} \hat{Q} \vec{\theta}-R_{1}(\vec{\theta})} d \vec{\theta}+O\left(\exp \left(-c n^{2 \varepsilon}\right)\right) \int_{\mathbb{R}^{n}} e^{-a_{n} \vec{\theta}^{T} \hat{Q} \vec{\theta}} d \vec{\theta}
\end{aligned}
$$

Using (8.6), we find that

$$
\begin{aligned}
& \int_{-n^{-1 / 2+\varepsilon}}^{n^{-1 / 2+\varepsilon}} \ldots \int_{-n^{-1 / 2+\varepsilon}}^{n^{-1 / 2+\varepsilon}}\left(\int_{-\frac{4}{\sigma} n^{-1 / 2+\varepsilon}} \phi_{1}^{4} e^{-a_{n} \vec{\theta}^{T} \hat{Q} \vec{\theta}-R_{1}(\vec{\theta})} d \theta_{1}\right) d \theta_{2} \ldots d \theta_{n} \\
= & \int_{-n^{-1 / 2+\varepsilon}}^{n^{-1 / 2+\varepsilon}} \ldots \int_{-n^{-1 / 2+\varepsilon}}^{n^{-1 / 2+\varepsilon}} e^{-a_{n} g_{1}\left(\theta_{2}, \ldots, \theta_{n}\right)-R_{2}(\vec{\theta})}\left(\int_{-\frac{4}{\sigma} n^{-1 / 2+\varepsilon}}^{\frac{4}{\sigma} n^{-1 / 2+\varepsilon}} \phi_{1}^{4} e^{-a_{n} \frac{\phi_{1}^{2}}{d_{1}+1}-8 b n \theta_{1}^{4}} d \theta_{1}\right) d \theta_{2} \ldots d \theta_{n},
\end{aligned}
$$

where $R_{2}(\vec{\theta})=8 n \sum_{j=2}^{n} \theta_{j}^{4}$. Using $(8.30)$, we get that as $n \rightarrow \infty$

$$
\int_{-\frac{4}{\sigma} n^{-1 / 2+\varepsilon}}^{\frac{4}{\sigma} n^{-1 / 2+\varepsilon}} \phi_{1}^{4} e^{-a_{n} \frac{\phi_{1}^{2}}{d_{1}+1}-8 b n \theta_{1}^{4}} d \theta_{1}=\left(1+O\left(\exp \left(-c n^{2 \varepsilon}\right)\right)\right) \int_{\left|\phi_{1}\right| \leq n^{1 / 2+\varepsilon}} \phi_{1}^{4} e^{-a_{n} \frac{\phi_{1}^{2}}{d_{1}+1}-8 b n \theta_{1}^{4}} d \theta_{1}
$$


Combining (8.38), (8.39), (8.40) and Lemma 8.1 with $x=\phi_{1} / n$, we obtain that as $n \rightarrow \infty$

$$
\begin{aligned}
\int_{U_{n}\left(n^{-1 / 2+\varepsilon}\right)} \phi_{1}^{4} e^{-a_{n} \vec{\theta}^{T} \hat{Q} \vec{\theta}-R_{1}(\vec{\theta})} d \vec{\theta} \leq & c^{\prime} n^{2} \int_{U_{n}\left(n^{-1 / 2+\varepsilon}\right)} e^{-a_{n} \vec{\theta}^{T} \hat{Q} \vec{\theta}-R_{1}(\vec{\theta})} d \vec{\theta} \\
& +O\left(\exp \left(-c n^{2 \varepsilon}\right)\right) \int_{\mathbb{R}^{n}} e^{-a_{n} \vec{\theta}^{T} \hat{Q} \vec{\theta}} d \vec{\theta}
\end{aligned}
$$

for some constants $c, c^{\prime}>0$ depending only on $a$ and $\sigma$.

Combining similar to (8.41) inequalities for $\phi_{1}, \phi_{2}, \ldots, \phi_{n}$ and using (8.33), we find that as $n \rightarrow \infty$

$$
\begin{array}{r}
\int_{U_{n}\left(n^{-1 / 2+\varepsilon}\right)} \theta_{1}^{4} e^{-a_{n} \vec{\theta}^{T} \hat{Q} \vec{\theta}-R_{1}(\vec{\theta})} d \vec{\theta} \leq \frac{\left(c_{\phi}+c_{\mu}\right) c^{\prime}}{n^{2}} \int_{U_{n}\left(n^{-1 / 2+\varepsilon}\right)} e^{-a_{n} \vec{\theta}^{T} \hat{Q} \vec{\theta}-R_{1}(\vec{\theta})} d \vec{\theta} \\
+O\left(\exp \left(-c n^{2 \varepsilon}\right)\right) \int_{\mathbb{R}^{n}} e^{-a_{n} \vec{\theta}^{T} \hat{Q} \vec{\theta}} d \vec{\theta}
\end{array}
$$

for some $c>0$ depending only on $a$ and $\sigma$. Note that as $n \rightarrow \infty$

$$
\begin{array}{r}
\int_{-n^{-1 / 2+\varepsilon}}^{n^{-1 / 2+\varepsilon}} \cdots \int_{-n^{-1 / 2+\varepsilon}}^{n^{-1 / 2+\varepsilon}} e^{-a_{n} g_{1}\left(\theta_{2}, \ldots, \theta_{n}\right)-R_{2}(\vec{\theta})}\left(\int_{-n^{-1 / 2+\varepsilon}}^{n^{-1 / 2+\varepsilon}} e^{-a_{n} \frac{\phi_{1}^{2}}{d_{1}+1}-8 b n \theta_{1}^{4}} d \theta_{1}\right) d \theta_{2} \ldots d \theta_{n} \\
=\cdots\left(\int_{-n^{-1 / 2+\varepsilon}}^{n^{-1 / 2+\varepsilon}} e^{-a_{n} \frac{\phi_{1}^{2}}{d_{1}+1}}\left(1-8 b n \theta_{1}^{4}+O\left(n^{-2+8 \varepsilon}\right)\right) d \theta_{1}\right) d \theta_{2} \ldots d \theta_{n}
\end{array}
$$

Combining (8.42) and (8.43), we get that as $n \rightarrow \infty$

$$
\begin{aligned}
\int_{U_{n}\left(n^{-1 / 2+\varepsilon}\right)} e^{-a_{n} \vec{\theta}^{T} \hat{Q} \vec{\theta}-R_{1}(\vec{\theta})} d \vec{\theta} \geq(1 & \left.+\frac{\tilde{c}}{n}\right) \int_{U_{n}\left(n^{-1 / 2+\varepsilon}\right)} e^{-a_{n} \vec{\theta}^{T} \hat{Q} \vec{\theta}-R_{2}(\vec{\theta})} d \vec{\theta} \\
& +O\left(\exp \left(-c n^{2 \varepsilon}\right)\right) \int_{\mathbb{R}^{n}} e^{-a_{n} \vec{\theta}^{T} \hat{Q} \vec{\theta}} d \vec{\theta}
\end{aligned}
$$

where $\tilde{c}$ depends only on $a, b$ and $\sigma$.

We continue similarly to $(8.44)$

$$
\begin{array}{r}
\int_{U_{n}\left(n^{-1 / 2+\varepsilon}\right)} e^{-a_{n} \vec{\theta}^{T} \hat{Q} \vec{\theta}-R_{k}(\vec{\theta})} d \vec{\theta} \geq\left(1+\frac{\tilde{c}}{n}\right) \int_{U_{n}\left(n^{-1 / 2+\varepsilon}\right)} e^{-a_{n} \vec{\theta}^{T} \hat{Q} \vec{\theta}-R_{k+1}(\vec{\theta})} d \vec{\theta} \\
+O\left(\exp \left(-c n^{2 \varepsilon}\right)\right) \int_{\mathbb{R}^{n}} e^{-a_{n} \vec{\theta}^{T} \hat{Q} \vec{\theta}} d \vec{\theta}
\end{array}
$$

where

$$
R_{k}(\vec{\theta})=8 n \sum_{j=k}^{n} \theta_{j}^{4}
$$


Combining all inequalities of (8.45) for $R_{1}, R_{2}, \ldots, R_{n}$, we get that

$$
\begin{aligned}
\int_{U_{n}\left(n^{-1 / 2+\varepsilon}\right)} e^{-a_{n} \vec{\theta}^{T} \hat{Q} \vec{\theta}-R_{1}(\vec{\theta})} d \vec{\theta} \geq & \left(1+\frac{\tilde{c}}{n}\right)^{n} \int_{U_{n}\left(n^{-1 / 2+\varepsilon}\right)} e^{-a_{n} \vec{\theta}^{T} \hat{Q} \vec{\theta}} d \vec{\theta} \\
& +O\left(\exp \left(-c n^{2 \varepsilon}\right)\right) \int_{\mathbb{R}^{n}} e^{-a_{n} \vec{\theta}^{T} \hat{Q} \vec{\theta}} d \vec{\theta}
\end{aligned}
$$

for some $c>0$ depending only on $a$ and $\sigma$. Note also that

$$
\int_{U_{n}\left(n^{-1 / 2+\varepsilon}\right)} e^{-a_{n} \vec{\theta}^{T} \hat{Q} \vec{\theta}-R_{1}(\vec{\theta})} d \vec{\theta} \leq \int_{U_{n}\left(n^{-1 / 2+\varepsilon}\right)} e^{-a_{n} \vec{\theta}^{T} \hat{Q} \vec{\theta}} d \vec{\theta}
$$

Combining (8.9), (8.47) and (8.48), we obtain (8.34).

Lemma 8.4. Let the assumptions of Lemma 3.2 hold. For positive constants $a, b, d_{1}, d_{2}$ let sequence of differentiable functions $\left\{R_{n}(\vec{\theta})\right\}$ be such that

$$
R e\left(R_{n}(\vec{\theta})\right) \leq d_{1} \frac{\vec{\theta}^{T} \hat{Q} \vec{\theta}}{n}
$$

and for $\vec{\theta} \in U_{n}\left(\frac{4}{\sigma} n^{-1 / 2+\varepsilon}\right)$

$$
\left|\frac{\partial R_{n}(\vec{\theta})}{\partial \theta_{k}}\right| \leq d_{2} n^{-1 / 2+3 \varepsilon}
$$

Then as $n \rightarrow \infty$

$$
\begin{array}{r}
\int_{U_{n}\left(n^{-1 / 2+\varepsilon}\right)} \phi_{k} e^{i \frac{b}{n} \phi_{k}-a \vec{\theta}^{T} \hat{Q} \vec{\theta}+R_{n}(\vec{\theta})} d \vec{\theta}=\Theta_{k_{1}, k_{2}}\left(\int_{U_{n}\left(n^{-3 / 2+\varepsilon}\right)} e^{i \frac{b}{n} \phi_{k}-a \vec{\theta}^{T} \hat{Q} \vec{\theta}+R_{n}(\vec{\theta})} d \vec{\theta}\right) \\
+O\left(n^{-1 / 2+4 \varepsilon}\right) \int_{\mathbb{R}^{n}} e^{-a \vec{\theta}^{T} \hat{Q} \vec{\theta}} d \vec{\theta}
\end{array}
$$

and

$$
\begin{aligned}
& \int_{U_{n}\left(n^{-1 / 2+\varepsilon}\right)} \phi_{k}^{2} e^{i \frac{b}{n} \phi_{k}-a \vec{\theta}^{T} \hat{Q} \vec{\theta}+R_{n}(\vec{\theta})} d \vec{\theta}=\Theta_{k_{1}, k_{2}}\left(n \int_{U_{n}\left(n^{-3 / 2+\varepsilon}\right)} e^{i \frac{b}{n} \phi_{k}-a \vec{\theta}^{T} \hat{Q} \vec{\theta}+R_{n}(\vec{\theta})} d \vec{\theta}\right) \\
& +O\left(\exp \left(-\tilde{c} n^{2 \varepsilon}\right)\right) \int_{\mathbb{R}^{n}} e^{-a \vec{\theta}^{T} \hat{Q} \vec{\theta}} d \vec{\theta},
\end{aligned}
$$

where constants $k_{1}, k_{2}, \tilde{c}>0$ depend only on $a, b, d_{1}, d_{2}$ and $\sigma$. 
Proof of Lemma 8.4. For our purpose it is convenient to assume that $k=1$. Note that

$$
\int_{U_{n}\left(n^{-1 / 2+\varepsilon}\right)}\left|e^{i \frac{b}{n} \phi_{1}-a \vec{\theta}^{T} \hat{Q} \vec{\theta}+R_{n}(\vec{\theta})}\right| d \vec{\theta} \leq \int_{U_{n}\left(n^{-1 / 2+\varepsilon}\right)} e^{-a \vec{\theta}^{T} \hat{Q} \vec{\theta}+d_{1} \frac{\vec{\theta}^{T} \hat{Q} \vec{\theta}}{n}} d \vec{\theta} .
$$

Using (8.2), we get that as $n \rightarrow \infty$

$$
\int_{U_{n}\left(n^{-1 / 2+\varepsilon}\right)}\left|e^{i \frac{b}{n} \phi_{1}-a \vec{\theta}^{T} \hat{Q} \vec{\theta}+R_{n}(\vec{\theta})}\right| d \vec{\theta}=O(1) \int_{U_{n}\left(n^{-1 / 2+\varepsilon}\right)} e^{-a \vec{\theta}^{T} \hat{Q} \vec{\theta}} d \vec{\theta} .
$$

Similar to (8.37), for $m=1,2$ we find that as $n \rightarrow \infty$

$$
\begin{aligned}
\int_{U_{n}\left(\frac{4}{\sigma} n^{-1 / 2+\varepsilon}\right)-U_{n}\left(n^{-1 / 2+\varepsilon}\right)} \phi_{1}^{m}\left|e^{i \frac{b}{n} \phi_{1}-a \vec{\theta}^{T} \hat{Q} \vec{\theta}+R_{n}(\vec{\theta})}\right| d \vec{\theta} & \\
= & O\left(\exp \left(-c n^{2 \varepsilon}\right)\right) \int_{\mathbb{R}^{n}} e^{-a \vec{\theta}^{T} \hat{Q} \vec{\theta}} d \vec{\theta}
\end{aligned}
$$

for some $c>0$ depending only on $a, d_{1}$ and $\sigma$. It follows that

$$
\begin{aligned}
& \int_{-n^{-1 / 2+\varepsilon}}^{n^{-1 / 2+\varepsilon}} \cdots \int_{-n^{-1 / 2+\varepsilon}}^{n^{-1 / 2+\varepsilon}}\left(\int_{-\frac{4}{\sigma} n^{-1 / 2+\varepsilon}}^{\frac{4}{\sigma} n^{-1 / 2+\varepsilon}} \phi_{1}^{m} e^{i \frac{b}{n} \phi_{1}-a \vec{\theta}^{T} \hat{Q} \vec{\theta}+R_{n}(\vec{\theta})} d \theta_{1}\right) d \theta_{2} \ldots d \theta_{n} \\
= & \int_{U_{n}\left(n^{-1 / 2+\varepsilon}\right)} \phi_{1}^{m} e^{i \frac{b}{n} \phi_{1}-a \vec{\theta}^{T} \hat{Q} \vec{\theta}+R_{n}(\vec{\theta})} d \vec{\theta}+O\left(\exp \left(-c n^{2 \varepsilon}\right)\right) \int_{\mathbb{R}^{n}} e^{-a \vec{\theta}^{T} \hat{Q} \vec{\theta}} d \vec{\theta}
\end{aligned}
$$

We define

$$
R_{n}^{\prime}(\vec{\theta})=R_{n}\left(0, \theta_{2}, \ldots, \theta_{n}\right)
$$

and

$$
r_{1}(\vec{\theta})=R_{n}(\vec{\theta})-R_{n}^{\prime}(\vec{\theta}) .
$$

According to Mean Value Theorem, for $\theta \in U_{n}\left(\frac{4}{\sigma} n^{-1 / 2+\varepsilon}\right)$ we have that

$$
\left|r_{1}(\vec{\theta})\right|=\left|R_{n}(\vec{\theta})-R_{n}^{\prime}(\vec{\theta})\right|=\left|\theta_{1} \frac{\partial R_{n}(\tilde{\vec{\theta}})}{\partial \theta_{k}}\right|=O\left(n^{-1+4 \varepsilon}\right) .
$$

Using (8.6), we find that

$$
\begin{aligned}
\int_{-n^{-1 / 2+\varepsilon}}^{n^{-1 / 2+\varepsilon}} & \ldots \int_{-n^{-1 / 2+\varepsilon}}^{n^{-1 / 2+\varepsilon}}\left(\int_{-\frac{4}{\sigma} n^{-1 / 2+\varepsilon}}^{\frac{4}{\sigma} n^{-1 / 2+\varepsilon}} \phi_{1}^{m} e^{i \frac{b}{n} \phi_{1}-a \vec{\theta}^{T} \hat{Q} \vec{\theta}+R_{n}(\vec{\theta})} d \theta_{1}\right) d \theta_{2} \ldots d \theta_{n} \\
& =\cdots \int_{-n^{-1 / 2+\varepsilon}}^{n^{-1 / 2+\varepsilon}} e^{-a g_{1}\left(\theta_{2}, \ldots, \theta_{n}\right)+R_{n}^{\prime}(\vec{\theta})}\left(\int_{-\frac{4}{\sigma} n^{-1 / 2+\varepsilon}}^{\frac{4}{\sigma} n^{-1 / 2+\varepsilon}} \phi_{1}^{m} e^{i \frac{b}{n} \phi_{1}-a \frac{\phi_{1}^{2}}{d_{1}+1}+r_{1}(\vec{\theta})} d \theta_{1}\right) d \theta_{2} \ldots d \theta_{n},
\end{aligned}
$$


Using (8.30), we get that as $n \rightarrow \infty$

$$
\begin{aligned}
& \int_{-\frac{4}{\sigma} n^{-1 / 2+\varepsilon}} \phi_{1}^{m} e^{i \frac{b}{n} n_{1}-a \frac{\phi_{1}^{2}}{d_{1}+1}+r_{1}(\vec{\theta})} d \theta_{1} \\
& =\left(1+O\left(\exp \left(-c n^{2 \varepsilon}\right)\right)\right) \int_{\left|\phi_{1}\right| \leq n^{1 / 2+\varepsilon}} \phi_{1}^{m} e^{i \frac{b}{n} \phi_{1}-a \frac{\phi_{1}^{2}}{d_{1}+1}+r_{1}(\vec{\theta})} d \theta_{1}
\end{aligned}
$$

Combining (8.56), (8.60), (8.61) with $m=2$ and (8.15) with $x=\phi_{1} / n$, we obtain (8.52).

Note that

$$
\begin{aligned}
& \int_{\left|\phi_{1}\right| \leq n^{1 / 2+\varepsilon}} \phi_{1} e^{i \frac{b}{n} \phi_{1}-a \frac{\phi_{1}^{2}}{d_{1}+1}+r_{1}(\vec{\theta})} d \theta_{1} \\
& =\int_{\left|\phi_{1}\right| \leq n^{1 / 2+\varepsilon}} \phi_{1}\left(1+i \frac{b}{n} \phi_{1}+O\left(n^{-1+2 \varepsilon}\right)\right) e^{-a \frac{\phi_{1}^{2}}{d_{1}+1}+r_{1}(\vec{\theta})} d \theta_{1}
\end{aligned}
$$

Since $\partial r_{1} / \partial \theta_{1}=\partial R_{n} / \partial \theta_{1}$, using (8.21) with $x=\phi_{1} / n$, we get that

$$
\int_{\left|\phi_{1}\right| \leq n^{1 / 2+\varepsilon}} \phi_{1} e^{-a \frac{\phi_{1}^{2}}{d_{1}+1}+r_{1}(\vec{\theta})} d \theta_{1}=O\left(n^{-1 / 2+4 \varepsilon}\right) \int_{\left|\phi_{1}\right| \leq n^{1 / 2+\varepsilon}} e^{-a \frac{\phi_{1}^{2}}{d_{1}+1}+r_{1}(\vec{\theta})} d \theta_{1}
$$

Combining (8.56), (8.60), (8.61) with $m=1$ and (8.52), (8.54) with $b=0$ and (8.63), we obtain (8.51).

Lemma 8.5. Let the assumptions of Lemma 3.2 hold. For positive constants $a, b, d_{1}, d_{2}$ let sequence of vectors $\left\{\vec{\beta}_{n}\right\}$ and sequence of real differentiable functions $\left\{R_{n}(\vec{\theta})\right\}$ be such that

$$
\begin{gathered}
\left\|\vec{\beta}_{n}\right\|_{\infty} \leq b, \\
R_{n}(\vec{\theta}) \leq d_{1} \frac{\vec{\theta}^{T} \hat{Q} \vec{\theta}}{n},
\end{gathered}
$$

and for $\vec{\theta} \in U_{n}\left(\frac{4}{\sigma} n^{-1 / 2+\varepsilon}\right)$

$$
\left\|\frac{\partial R_{n}(\vec{\theta})}{\partial \vec{\theta}}\right\|_{\infty} \leq d_{2} n^{-1 / 2+3 \varepsilon}
$$

Then as $n \rightarrow \infty$

$$
\begin{array}{r}
\int_{U_{n}\left(n^{-1 / 2+\varepsilon}\right)} e^{i \vec{\beta}_{n}^{T} \vec{\theta}-a \vec{\theta}^{T} \hat{Q} \vec{\theta}+R_{n}(\vec{\theta})} d \vec{\theta}=\Theta_{\tilde{k}_{1}, \tilde{k}_{2}}\left(\int_{U_{n}\left(n^{-1 / 2+\varepsilon}\right)} e^{-a \vec{\theta}^{T} \hat{Q} \vec{\theta}+\tilde{R}_{n}(\vec{\theta})} d \vec{\theta}\right) \\
+O\left(n^{-1 / 2+4 \varepsilon}\right) \int_{\mathbb{R}^{n}} e^{-a \vec{\theta}^{T} \hat{Q} \vec{\theta}} d \vec{\theta},
\end{array}
$$


where $\tilde{R}_{n}=R_{n}-\frac{1}{2} \sum_{j=1}^{n} \beta_{j}^{2} \theta_{j}^{2}$ and constants $\tilde{k}_{1}, \tilde{k}_{2}$ depend only on $a, b, d_{1}, d_{2}$ and $\sigma$.

Proof of Lemma 8.5. Using (8.54), we get that as $n \rightarrow \infty$

$$
\begin{aligned}
& \int_{U_{n}\left(n^{-1 / 2+\varepsilon}\right)} e^{i \vec{\beta}_{n}^{T} \vec{\theta}-a \vec{\theta}^{T} \hat{Q} \vec{\theta}+R_{n}(\vec{\theta})} d \vec{\theta} \\
= & \int_{U_{n}\left(n^{-1 / 2+\varepsilon}\right)}\left(1+i \beta_{1} \theta_{1}-\frac{\beta_{1}^{2} \theta_{1}^{2}}{2}+O\left(n^{-3 / 2+3 \varepsilon}\right)\right) e^{i\left(\vec{\beta}_{n}^{T} \vec{\theta}-\beta_{1} \theta_{1}\right)-a \vec{\theta}^{T} \hat{Q} \vec{\theta}+R_{n}(\vec{\theta})} d \vec{\theta} \\
= & \int_{U_{n}\left(n^{-1 / 2+\varepsilon}\right)} e^{i\left(\vec{\beta}_{n}^{T} \vec{\theta}-\beta_{1} \theta_{1}\right)-a \vec{\theta}^{T} \hat{Q} \vec{\theta}+R_{n}(\vec{\theta})-\frac{1}{2} \beta_{1}^{2} \theta_{1}^{2}} d \vec{\theta}+O\left(n^{-3 / 2+3 \varepsilon}\right) \int_{U_{n}\left(n^{-1 / 2+\varepsilon}\right)} e^{-a \vec{\theta}^{T} \hat{Q} \vec{\theta}} d \vec{\theta} \\
& +\int_{U_{n}\left(n^{-1 / 2+\varepsilon}\right)} i \beta_{1} \theta_{1} e^{i\left(\vec{\beta}_{n}^{T} \vec{\theta}-\beta_{1} \theta_{1}\right)-a \vec{\theta}^{T} \hat{Q} \vec{\theta}+R_{n}(\vec{\theta})} d \vec{\theta} .
\end{aligned}
$$

Taking into account (8.28) and using Lemma 8.4, we find that as $n \rightarrow \infty$

$$
\begin{aligned}
& \int_{U_{n}\left(n^{-1 / 2+\varepsilon}\right)} \phi_{k} e^{i\left(\vec{\beta}_{n}^{T} \vec{\theta}-\beta_{1} \theta_{1}\right)-a \vec{\theta}^{T} \hat{Q} \vec{\theta}+R_{n}(\vec{\theta})} d \vec{\theta} \\
= & \Theta_{k_{1}, k_{2}}\left(\int_{U_{n}\left(n^{-3 / 2+\varepsilon}\right)} e^{i\left(\vec{\beta}_{n}^{T} \vec{\theta}-\beta_{1} \theta_{1}\right)-a \vec{\theta}^{T} \hat{Q} \vec{\theta}+R_{n}(\vec{\theta})} d \vec{\theta}\right)+O\left(n^{-1 / 2+4 \varepsilon}\right) \int_{\mathbb{R}^{n}} e^{-a \vec{\theta}^{T} \hat{Q} \vec{\theta}} d \vec{\theta} \\
= & \Theta_{k_{1}, k_{2}}\left(\int_{U_{n}\left(n^{-3 / 2+\varepsilon}\right)} e^{i\left(\vec{\beta}_{n}^{T} \vec{\theta}-\beta_{1} \theta_{1}\right)-a \vec{\theta}^{T} \hat{Q} \vec{\theta}+R_{n}(\vec{\theta})-\frac{1}{2} \beta_{1}^{2} \theta_{1}^{2}} d \vec{\theta}\right)+O\left(n^{-1 / 2+4 \varepsilon}\right) \int_{\mathbb{R}^{n}} e^{-a \vec{\theta}^{T} \hat{Q} \vec{\theta}} d \vec{\theta},
\end{aligned}
$$

where constants $k_{1}, k_{2}$ depend only on $a, b, d_{1}, d_{2}$ and $\sigma$.

According to Lemma 3.2, we have that

$$
\left\|\hat{Q}^{-1}\right\|_{1}=\left\|\hat{Q}^{-1}\right\|_{\infty} \leq \frac{c_{\infty}}{n}
$$

Thus as $n \rightarrow \infty$

$$
\begin{aligned}
& \left|\int_{U_{n}\left(n^{-1 / 2+\varepsilon}\right)} \theta_{k} e^{i\left(\vec{\beta}_{n}^{T} \vec{\theta}-\beta_{1} \theta_{1}\right)-a \vec{\theta}^{T} \hat{Q} \vec{\theta}+R_{n}(\vec{\theta})} d \vec{\theta}\right| \\
& \leq \frac{\tilde{c}}{n}\left|\int_{U_{n}\left(n^{-3 / 2+\varepsilon}\right)} e^{i\left(\vec{\beta}_{n}^{T} \vec{\theta}-\beta_{1} \theta_{1}\right)-a \vec{\theta}^{T} \hat{Q} \vec{\theta}+R_{n}(\vec{\theta})-\frac{1}{2} \beta_{1}^{2} \theta_{1}^{2}} d \vec{\theta}\right|+O\left(n^{-3 / 2+4 \varepsilon}\right) \int_{\mathbb{R}^{n}} e^{-a \vec{\theta}^{T} \hat{Q} \vec{\theta}} d \vec{\theta},
\end{aligned}
$$


where $\tilde{c}>0$ depends only on $a, b, d_{1}, d_{2}$ and $\sigma$.

Combining (8.68) and (8.71), we get that as $n \rightarrow \infty$

$$
\begin{gathered}
\int_{U_{n}\left(n^{-1 / 2+\varepsilon}\right)} e^{i \vec{\beta}_{n}^{T} \vec{\theta}-a \vec{\theta}^{T} \hat{Q} \vec{\theta}+R_{n}(\vec{\theta})} d \vec{\theta} \\
=\left(1+\frac{c^{(1)}}{n}\right) \int_{U_{n}\left(n^{-3 / 2+\varepsilon}\right)} e^{i\left(\vec{\beta}_{n}^{T} \vec{\theta}-\beta_{1} \theta_{1}\right)-a \vec{\theta}^{T} \hat{Q} \vec{\theta}+R_{n}(\vec{\theta})-\frac{1}{2} \beta_{1}^{2} \theta_{1}^{2}} d \vec{\theta} \\
+O\left(n^{-3 / 2+4 \varepsilon}\right) \int_{\mathbb{R}^{n}} e^{-a \vec{\theta}^{T} \hat{Q} \vec{\theta}} d \vec{\theta}
\end{gathered}
$$

where $\left|c^{(1)}\right| \leq \tilde{c} \beta_{1} \leq \tilde{c} b$.

We define

$$
R_{n}^{(k)}=R_{n}-\frac{1}{2} \sum_{j=1}^{k} \beta_{j}^{2} \theta_{j}^{2} .
$$

We continue similarly to $(8.72)$

$$
\begin{gathered}
\int_{U_{n}\left(n^{-1 / 2+\varepsilon}\right)} e^{i\left(\vec{\beta}_{n}^{T} \vec{\theta}-\sum_{j=1}^{k} \beta_{j} \theta_{j}\right)-a \vec{\theta}^{T} \hat{Q} \vec{\theta}+R_{n}^{(k)}(\vec{\theta})} d \vec{\theta} \\
=\left(1+\frac{c^{(k+1)}}{n}\right) \int_{U_{n}\left(n^{-3 / 2+\varepsilon}\right)} e^{i\left(\vec{\beta}_{n}^{T} \vec{\theta}-\sum_{j=1}^{k+1} \beta_{j} \theta_{j}\right)-a \vec{\theta}^{T} \hat{Q} \vec{\theta}+R_{n}^{(k+1)}(\vec{\theta})} d \vec{\theta} \\
+O\left(n^{-3 / 2+4 \varepsilon}\right) \int_{\mathbb{R}^{n}} e^{-a \vec{\theta}^{T} \hat{Q} \vec{\theta}} d \vec{\theta},
\end{gathered}
$$

where $\left|c^{(k+1)}\right| \leq \tilde{c} \beta_{k} \leq \tilde{c} b$.

Combining all inequalities of (8.74) for $k=0,1, \ldots, n-1$, we get that as $n \rightarrow \infty$

$$
\begin{aligned}
& \int_{U_{n}\left(n^{-1 / 2+\varepsilon}\right)} e^{i \vec{\beta}_{n}^{T} \vec{\theta}-a \vec{\theta}^{T} \hat{Q} \vec{\theta}+R_{n}(\vec{\theta})} d \vec{\theta} \\
& =\left(1+\frac{c^{(1)}}{n}\right) \cdots\left(1+\frac{c^{(n)}}{n}\right) \int_{U_{n}\left(n^{-3 / 2+\varepsilon}\right)} e^{-a \vec{\theta}^{T} \hat{Q} \vec{\theta}+\tilde{R}_{n}(\vec{\theta})} d \vec{\theta} \\
& \\
& \quad+O\left(n^{-1 / 2+4 \varepsilon}\right) \int_{\mathbb{R}^{n}} e^{-a \vec{\theta}^{T} \hat{Q} \vec{\theta}} d \vec{\theta} .
\end{aligned}
$$

Since $\left|c^{(k)}\right| \leq b \tilde{c}$ for $k=0,1, \ldots, n-1$, using (8.75), we obtain (8.67). 
Proof of Lemma 5.2. Note that for $\vec{\theta} \in U_{n}\left(\frac{4}{\sigma} n^{-1 / 2+\varepsilon}\right)$ as $n \rightarrow \infty$

$$
\left\|\frac{\partial}{\partial \vec{\theta}} \sum_{\left(v_{j}, v_{k}\right) \in E G} \Delta_{j k}^{4}\right\|_{\infty}=O\left(n^{-1 / 2+3 \varepsilon}\right) .
$$

We define $\vec{\beta}_{n}=Q \overrightarrow{\alpha_{n}}$. Using Lemma 8.5, we find that as $n \rightarrow \infty$

$$
\begin{array}{r}
\int_{U_{n}\left(n^{-1 / 2+\varepsilon)}\right.} \exp \left(i \vec{\theta}^{T} Q \overrightarrow{\alpha_{n}}-a \vec{\theta}^{T} \hat{Q} \vec{\theta}-b \sum_{\left(v_{j}, v_{k}\right) \in E G} \Delta_{j k}^{4}+R_{n}(\vec{\theta})\right) d \vec{\theta} \\
=\Theta_{\tilde{k}_{1}, \tilde{k}_{2}}\left(\int_{U_{n}\left(n^{-1 / 2+\varepsilon)}\right.} \exp \left(-a \vec{\theta}^{T} \hat{Q} \vec{\theta}-b \sum_{\left(v_{j}, v_{k}\right) \in E G} \Delta_{j k}^{4}+\tilde{R}_{n}(\vec{\theta})\right) d \vec{\theta}\right) \\
+O\left(n^{-1 / 2+4 \varepsilon}\right) \int_{\mathbb{R}^{n}} e^{-a \vec{\theta}^{T} \hat{Q} \vec{\theta}},
\end{array}
$$

where $\tilde{R}_{n}=R_{n}-\frac{1}{2} \sum_{j=1}^{n} \beta_{j}^{2} \theta_{j}^{2}$ and constants $\tilde{k}_{1}, \tilde{k}_{2}$ depend only on $a, b, d_{1}, d_{2}$ and $\sigma$. Note that for some $d_{3}>0$, depending only on $c$ and $\sigma$,

$$
\frac{1}{2} \sum_{j=1}^{n} \beta_{j}^{2} \theta_{j}^{2} \leq d_{3} \frac{\vec{\theta}^{T} \hat{Q} \vec{\theta}}{n}
$$

Combining (8.2), (8.77) and Lemma 8.3, we find that as $n \rightarrow \infty$

$$
\begin{array}{r}
\int_{U_{n}\left(n^{-1 / 2+\varepsilon}\right)} \exp \left(i \vec{\theta}^{T} Q \overrightarrow{\alpha_{n}}-a \vec{\theta}^{T} \hat{Q} \vec{\theta}-b \sum_{\left(v_{j}, v_{k}\right) \in E G} \Delta_{j k}^{4}+R_{n}(\vec{\theta})\right) d \vec{\theta} \\
=\Theta_{k_{1}^{\prime}, k_{2}^{\prime}}\left(2^{\frac{n-1}{2}} \pi^{\frac{n-1}{2}} / \sqrt{\operatorname{det} \hat{Q}}\right),
\end{array}
$$

where constants $k_{1}^{\prime}, k_{2}^{\prime}$ depend only on $a, b, d_{1}, d_{2}$ and $\sigma$. Note that

$$
\begin{array}{r}
\int_{-\infty}^{+\infty} e^{-a n x^{2}} d x \int_{L \cap V_{0}} \exp \left(i \vec{\theta}^{T} Q \overrightarrow{\alpha_{n}}-a \sum_{\left(v_{j}, v_{k}\right) \in E G} \Delta_{j k}^{2}-b \sum_{\left(v_{j}, v_{k}\right) \in E G} \Delta_{j k}^{4}+R_{n}(\vec{\theta})\right) d L \\
=\int_{P(\theta) \in L \cap V_{0}} \exp \left(i \overrightarrow{\vec{\theta}^{T}} Q \overrightarrow{\alpha_{n}}-a \vec{\theta}^{T} \hat{Q} \vec{\theta}-b \sum_{\left(v_{j}, v_{k}\right) \in E G} \Delta_{j k}^{4}+R_{n}(\vec{\theta})\right) d \vec{\theta} .
\end{array}
$$

We have that

$$
\left|\exp \left(i \overrightarrow{\theta^{T}} \overrightarrow{Q \alpha_{n}}-a \vec{\theta}^{T} \hat{Q} \vec{\theta}-b \sum_{\left(v_{j}, v_{k}\right) \in E G} \Delta_{j k}^{4}+R_{n}(\vec{\theta})\right)\right| \leq e^{-a \vec{\theta}^{T} \hat{Q} \vec{\theta}+\frac{d_{1}}{n} \vec{\theta}^{T} \hat{Q} \vec{\theta}}
$$


Thus, combining (8.9), (8.12), (8.79) and (8.80), we obtain (5.33)

\section{$9 \quad$ Final remarks}

In fact, using Lemma 5.1 and Lemma 6.3, the estimation of the number of Eulerian circuits is reduced (see proof of Lemma 5.3) to estimating the integral

$$
\int_{V_{0}} \exp \left(i \vec{\theta}^{T} Q \vec{\alpha}-\frac{1}{2} \sum_{\left(v_{j}, v_{k}\right) \in E G} \Delta_{j k}^{2}-\frac{1}{12} \sum_{\left(v_{j}, v_{k}\right) \in E G} \Delta_{j k}^{4}+\operatorname{tr}\left(\Lambda \hat{Q}^{-1}\right)^{2}\right) d \vec{\theta},
$$

where $\alpha$ denotes the vector composed of the diagonal elements of $\hat{Q}^{-1}, \Lambda$ denotes the diagonal matrix whose diagonal elements are equal to components of the vector $Q \vec{\theta}$. Apparently, it is possible to estimate integral (9.1) more accurately for particular classes of graphs and obtain asymptotic formulas for $\operatorname{Eul}(G)$, similar to (2.12).

Finally, we want to note that the following expression

$$
2^{|E G|-\frac{n-1}{2}} \pi^{-\frac{n-1}{2}} \sqrt{t(G)} \prod_{j=1}^{n}\left(\frac{d_{j}}{2}-1\right) !
$$

gives a surprisingly good estimate for the number of Eulerian circuits in graphs. Namely, we calculated the exact numbers of Eulerian circuits for small random graphs and in all cases the values given by (9.2) differ from the exact ones within not more than $30 \%$ error.

\section{References}

[1] T. van Aardenne-Ehrenfest, N.G. de Bruijn, Circuits and trees in oriented linear graphs, Simon Stevin, 1951, 28, 203-217.

[2] N. L. Biggs, E. K. Lloyd and R. J. Wilson, Graph Theory, 1736-1936, Clarendon Press, Oxford, 1976.

[3] G. Brightwell, P. Winkler, Note on Counting Eulerian Circuits, Proceedings of the 7th ALENEX and 2nd ANALCO 2005 , ALENEX/ANALCO 2005 Vancouver, BC, C Demetrescu, R Sedgewick and R Tamassia (eds.), (2005) 259-262. arXiv:cs/0405067v1.

[4] M. Fiedler, Algebraic connectivity of graphs, Czech. Math. J. 23 (98) (1973), 298-305.

[5] G. Kirchhoff, Über die Auflösung der Gleichungen, auf welche man bei der Untersuchung der linearen Verteilung galvanischer Ströme geführt wird, Ann. Phys. Chem. 72 (1847), 497-508. Translated by J. B. O'Toole in I.R.E. Trans. Circuit Theory, CT-5 (1958) 4.

[6] B. D. McKay, R. W. Robinson, Asymptotic enumeration of eulerian circuits in the complete graph. Combinatorica, 7(4), December 1998. 
[7] B. Mohar, The Laplacian spectrum of graphs, Graph Theory, Combinatorics, and Applications, Vol. 2, Ed. Y. Alavi, G. Chartrand, O. R. Oellermann, A. J. Schwenk, Wiley, 1991, pp. 871-898.

[8] C. A. B. Smith and W. T. Tutte, On unicursal paths in a network of degree 4, Amer.Math. Monthly, 48 (1941) 233-237.

[9] W. T. Tutte, The dissection of equilateral triangles into equilateral triangles, Proc. Cambridge Philos. Soc., 44 (1948), 463482. 